\title{
Light baryon masses with dynamical twisted mass fermions
}

\author{
C. Alexandrou, ${ }^{1}$ R. Baron, ${ }^{2}$ B. Blossier, ${ }^{3}$ M. Brinet,${ }^{4}$ J. Carbonell,,${ }^{4}$ P. Dimopoulos, ${ }^{5}$ V. Drach, ${ }^{4}$ F. Farchioni, ${ }^{6}$ \\ R. Frezzotti, ${ }^{5}$ P. Guichon, ${ }^{2}$ G. Herdoiza, ${ }^{3}$ K. Jansen, ${ }^{3}$ T. Korzec,,${ }^{1}$ G. Koutsou, ${ }^{1}$ Z. Liu, ${ }^{8}$ C. Michael, ${ }^{7}$ O. Pène, ${ }^{8}$ A. Shindler, ${ }^{7}$ \\ C. Urbach, ${ }^{10}$ and U. Wenger ${ }^{9}$ \\ ${ }^{1}$ Department of Physics, University of Cyprus, P.O. Box 20537, 1678 Nicosia, Cyprus \\ ${ }^{2}$ CEA-Saclay, IRFU/Service de Physique Nucléaire, 91191 Gif-sur-Yvette, France \\ ${ }^{3}$ NIC, DESY, Zeuthen, Platanenallee 6, D-15738 Zeuthen, Germany \\ ${ }^{4}$ Laboratoire de Physique Subatomique et Cosmologie, UJF/CNRS/IN ${ }_{2} P_{3}, 53$ avenue des Martyrs, 38026 Grenoble, France \\ ${ }^{5}$ Dip. di Fisica, Università di Roma Tor Vergata and INFN, Sez. di Roma Tor Vergata, Via della Ricerca Scientifica, \\ I-00133 Roma, Italy \\ ${ }^{6}$ Universität Münster, Institut für Theoretische Physik, Wilhelm-Klemm-Strasse 9, D-48149 Münster, Germany \\ ${ }^{7}$ Theoretical Physics Division, Department of Mathematical Sciences, University of Liverpool, Liverpool L69 7ZL, United Kingdom \\ ${ }^{8}$ Laboratoire de Physique Théorique (Bât. 210), Université de Paris XI, CNRS-UMR8627, \\ Centre d'Orsay, 91405 Orsay-Cedex, France \\ ${ }^{9}$ Institute for Theoretical Physics, ETH Zürich, CH-8093 Zürich, Switzerland \\ ${ }^{10}$ Humboldt Universität zu Berlin, Fachbereich Physik, Inst. fur Elementarteilchenphysik, Newtonstr. 15, D-12489 Berlin, Germany
} (Received 21 March 2008; published 22 July 2008)

\begin{abstract}
We present results on the mass of the nucleon and the $\Delta$ using two dynamical degenerate twisted mass quarks and the tree-level Symanzik improved gauge action. The evaluation is performed at four quark masses corresponding to a pion mass in the range of about 300-600 MeV on lattices of 2.1-2.7 fm at three lattice spacings less than $0.1 \mathrm{fm}$. We check for cutoff effects by evaluating these baryon masses on lattices of spatial size $2.1 \mathrm{fm}$ at $\beta=3.9$ and $\beta=4.05$ and on a lattice of $2.4 \mathrm{fm}$ at $\beta=3.8$. The values we find are compatible within our statistical errors. Lattice results are extrapolated to the physical limit using continuum chiral perturbation theory. Performing a combined fit to our lattice data at $\beta=3.9$ and $\beta=$ 4.05 we find a nucleon mass of $963 \pm 12$ (stat) \pm 8 (syst) $\mathrm{MeV}$ where we used the lattice spacings determined from the pion decay constant to convert to physical units. The systematic error due to the chiral extrapolation is estimated by comparing results obtained at $\mathcal{O}\left(p^{3}\right)$ and $\mathcal{O}\left(p^{4}\right)$ heavy baryon chiral perturbation theory. The nucleon mass at the physical point provides an independent determination of the lattice spacing. Using heavy baryon chiral perturbation theory at $\mathcal{O}\left(p^{3}\right)$ we find $a_{\beta=3.9}=0.0889 \pm$ 0.0012 (stat) \pm 0.0014 (syst) fm, and $a_{\beta=4.05}=0.0691 \pm 0.0010$ (stat) \pm 0.0010 (syst) fm, in good agreement with the values determined from the pion decay constant. Using results from our two smaller lattices spacings at constant $r_{0} m_{\pi}$ we estimate the continuum limit and check consistency with results from the coarser lattice. Results at the continuum limit are chirally extrapolated to the physical point. Isospin violating lattice artifacts in the $\Delta$-system are found to be compatible with zero for the values of the lattice spacings used in this work. Performing a combined fit to our lattice data at $\beta=3.9$ and $\beta=4.05$ we find for the masses of the $\Delta^{++,-}$and $\Delta^{+, 0} 1315 \pm 24$ (stat) MeV and $1329 \pm 30$ (stat) MeV, respectively. We confirm that in the continuum limit they are also degenerate.
\end{abstract}

DOI: 10.1103/PhysRevD.78.014509

PACS numbers: 11.15.Ha, 12.38.-t, 12.38.Aw, 12.38.Gc

\section{INTRODUCTION}

Twisted mass fermions provide an attractive formulation of lattice QCD that allows for automatic $\mathcal{O}(a)$ improvement, infrared regularization of small eigenvalues, and fast dynamical simulations [1-5]. A particularly attractive feature is that automatic $\mathcal{O}(a)$ improvement is obtained by tuning only one parameter requiring no further improvements on the operator level. A tree-level analysis of cutoff effects for twisted mass fermions has been presented in Ref. [6], while a preliminary nonperturbative investigation on scaling of several observables is carried out in Ref. [7]. Recent simulations with two degenerate flavors of dynamical Wilson twisted mass fermions demonstrate that pion masses of $m_{\pi} \gtrsim 300 \mathrm{MeV}$ can be reached using Hybrid Monte Carlo methods [5,8,9]. The theoretical framework to include the strange and charm quarks has been laid out and practical simulations are being investigated [10-12]. Important physical results are emerging using gauge configurations generated with two degenerate twisted quarks: In the meson sector very precise results on the pion mass and decay constant led to the determination of the low energy constants $\bar{l}_{3}, \bar{l}_{4}, \mathrm{~F}$, and $B_{0}[7,13,14]$ to an accuracy that had an immediate impact on chiral perturbation theory $(\chi \mathrm{PT})$ predictions [15]. Accurate results on the pion form factor are obtained [16] using the "one-end-trick" method developed in Refs. $[17,18]$. The kaon system is studied in a partially quenched approach by implementing nondegen- 
erate valence twisted mass quarks maintaining automatic $\mathcal{O}(a)$ improvement [19-22]. After determining the average up and down quark mass and the strange quark mass, the kaon decay constant is extracted [23,24]. In a similar approach first results on the charm quark mass and decay constant are obtained [25]. Preliminary results on the first moment of the pion quark distribution function were reported in Ref. [26].

In this work we present a detailed analysis of results in the light baryon sector, a subset of which was given in Ref. [27]. Using two dynamical degenerate twisted mass quarks we evaluate the mass of the nucleon and $\Delta$ for pion masses down to about $300 \mathrm{MeV}$. We use the tree-level Symanzik improved gauge action [28]. We perform the calculation using three different lattice spacings corresponding to $\beta=3.8, \beta=3.9$, and $\beta=4.05$ to check cutoff effects, where $\beta \equiv 6 / g^{2}$ with $g$ being the bare coupling constant. For each value of $\beta$ we have configurations at four different values of the bare quark mass chosen so that the pion masses are in the range of about $300 \mathrm{MeV}$ to $600 \mathrm{MeV}$. These gauge configurations belong to the same ensembles as those analyzed for the evaluation of the pion mass and decay constant. The values of the lattice spacing extracted from the pion decay constant are $a_{\beta=3.8}=0.0995(7) \mathrm{fm}, \quad a_{\beta=3.9}=0.0855(5) \mathrm{fm}, \quad$ and $a_{\beta=4.05}=0.0667(5) \mathrm{fm}[13,14]$ and will be used in this work. At $\beta=3.9$, for the smallest pion mass, there are gauge configurations at two different volumes enabling us to assess finite volume effects.

Chiral perturbation theory has been successfully applied in the extrapolation of lattice data obtained with twisted mass fermions in the pion sector yielding an accurate determination of the relevant low energy constants. Applying $\chi \mathrm{PT}$ to the baryon sector is more involved and several variants exist. However, to leading one-loop order, the result is well established and the quality of our lattice results allows for extrapolation to the physical point using this lowest order result. Performing a combined fit to our lattice data at $\beta=3.9$ and $\beta=4.05$ using the leading oneloop order result we find a nucleon mass of $963 \pm$ 12 (stat) $\mathrm{MeV}$, where we convert to physical units using the lattice spacing determined from $f_{\pi}$. We would like to point out that in most other chiral extrapolations of lattice data the physical point is included in the fits and therefore such a consistency check cannot be made. The nucleon mass at the physical point provides an independent determination of the lattice spacing. We find that the lattice spacing thus determined is in good agreement with the value extracted in the pion sector. This is a nontrivial check of our lattice formulation and of the smallness of the systematic errors involved. To assess systematic errors due to the chiral extrapolation we perform chiral fits to the nucleon and $\Delta$ mass using higher order chiral perturbation theory results, which also include explicitly the $\Delta$ degree of freedom.
One of the drawbacks of twisted mass fermions is the $\mathcal{O}\left(a^{2}\right)$ breaking of isospin symmetry, which is only restored in the continuum limit. In the baryon sector we can study isospin breaking by evaluating the mass difference between $\Delta^{++}\left(\Delta^{-}\right)$and $\Delta^{+}\left(\Delta^{0}\right)$. Unlike in the pion sector, where disconnected contributions enter in the evaluation of the mass of the $\pi^{0}$, here there are none. We can therefore obtain an accurate evaluation of isospin splitting and its dependence on the lattice spacing. We find no isospin splitting within our statistical accuracy. This is in agreement with a theoretical analysis $[29,30]$ that shows potentially large $\mathcal{O}\left(a^{2}\right)$ flavor breaking effects to appear in the $\pi^{0}$-mass but to be suppressed in other quantities. Like in the nucleon case, we perform a combined fit to our lattice data at $\beta=3.9$ and $\beta=4.05$ for the mass of the $\Delta^{++,-}$and $\Delta^{+, 0}$ using the lowest one-loop order chiral perturbation result. We find for the mass of the $\Delta^{++,-}$and $\Delta^{+, 0} 1315 \pm 24$ (stat) $\mathrm{MeV}$ and $1329 \pm 30$ (stat) MeV, respectively. We confirm that in the continuum limit they are also degenerate.

This paper is organized as follows: In Sec. II we present our lattice action and in Sec. III we explain our lattice techniques. In Sec. IV we discuss lattice artifacts and in Sec. V we give results on the nucleon and $\Delta$ mass and also describe the chiral extrapolations. Finally, in Sec. VI we provide a summary and conclusions.

\section{LATTICE ACTION}

For the gauge fields we use the tree-level Symanzik improved gauge action [28], which includes besides the plaquette term $U_{x, \mu, \nu}^{1 \times 1}$ also rectangular $(1 \times 2)$ Wilson loops $U_{x, \mu, \nu}^{1 \times 2}$

$$
\begin{aligned}
S_{g}= & \frac{\beta}{3} \sum_{x}\left(b_{0} \sum_{\substack{\mu, \nu=1 \\
1 \leq \mu<\nu}}^{4}\left\{1-\operatorname{Re} \operatorname{Tr}\left(U_{x, \mu, \nu}^{1 \times 1}\right)\right\}\right. \\
& \left.+b_{1} \sum_{\substack{\mu, \nu=1 \\
\mu \neq \nu}}^{4}\left\{1-\operatorname{Re} \operatorname{Tr}\left(U_{x, \mu, \nu}^{1 \times 2}\right)\right\}\right)
\end{aligned}
$$

with $b_{1}=-1 / 12$ and the (proper) normalization condition $b_{0}=1-8 b_{1}$. Note that at $b_{1}=0$ this action becomes the usual Wilson plaquette gauge action.

The fermionic action for two degenerate flavors of quarks in twisted mass QCD is given by

$$
S_{F}=a^{4} \sum_{x} \bar{\chi}(x)\left(D_{W}[U]+m_{0}+i \mu \gamma_{5} \tau^{3}\right) \chi(x)
$$

with $\tau^{3}$ the Pauli matrix acting in the isospin space, $\mu$ the bare twisted mass and the massless Wilson-Dirac operator given by

$$
D_{W}[U]=\frac{1}{2} \gamma_{\mu}\left(\nabla_{\mu}+\nabla_{\mu}^{*}\right)-\frac{a r}{2} \nabla_{\mu} \nabla_{\mu}^{*},
$$

where 


$$
\begin{aligned}
& \nabla_{\mu} \psi(x)=\frac{1}{a}\left[U_{\mu}^{\dagger}(x) \psi(x+a \hat{\mu})-\psi(x)\right] \text { and } \\
& \nabla_{\mu}^{*} \psi(x)=-\frac{1}{a}\left[U_{\mu}(x-a \hat{\mu}) \psi(x-a \hat{\mu})-\psi(x)\right] .
\end{aligned}
$$

Maximally twisted Wilson quarks are obtained by setting the untwisted quark mass $m_{0}$ to its critical value $m_{\mathrm{cr}}$, while the twisted quark mass parameter $\mu$ is kept nonvanishing in order to work away from the chiral limit. In Eq. (2) the quark fields $\chi$ are in the so-called "twisted basis." The "physical basis" is obtained for maximal twist by the simple transformation

$$
\begin{aligned}
& \psi(x)=\exp \left(\frac{i \pi}{4} \gamma_{5} \tau^{3}\right) \chi(x), \\
& \bar{\psi}(x)=\bar{\chi}(x) \exp \left(\frac{i \pi}{4} \gamma_{5} \tau^{3}\right) .
\end{aligned}
$$

In terms of the physical fields the action is given by

$$
\begin{aligned}
S_{F}^{\psi}= & a^{4} \sum_{x} \bar{\psi}(x)\left(\frac{1}{2} \gamma_{\mu}\left[\nabla_{\mu}+\nabla_{\mu}^{*}\right]\right. \\
& \left.-i \gamma_{5} \tau^{3}\left(-\frac{a r}{2} \nabla_{\mu} \nabla_{\mu}^{*}+m_{\mathrm{cr}}\right)+\mu\right) \psi(x) .
\end{aligned}
$$

In this paper, unless otherwise stated, the quark fields will be understood as "physical fields," $\psi$, in particular, when we define the baryonic interpolating fields.

A crucial advantage of the twisted mass formulation is the fact that, by tuning the bare untwisted quark mass $m_{0}$ to its critical value $m_{\mathrm{cr}}$, all physical observables are automatically $\mathcal{O}(a)$ improved. In practice, we implement maximal twist of Wilson quarks by tuning to zero the bare untwisted current quark mass, commonly called PCAC mass, $m_{\mathrm{PCAC}}$, which is proportional to $m_{0}-m_{\text {cr }}$ up to $\mathcal{O}(a)$ corrections. As detailed in Ref. [31], $m_{\mathrm{PCAC}}$ is conveniently evaluated through

$$
m_{\mathrm{PCAC}}=\lim _{t / a \gg 1} \frac{\sum_{\mathbf{x}}\left\langle\partial_{4} \tilde{A}_{4}^{b}(\mathbf{x}, t) \tilde{P}^{b}(0)\right\rangle}{2 \sum_{\mathbf{x}}\left\langle\tilde{P}^{b}(\mathbf{x}, t) \tilde{P}^{b}(0)\right\rangle}, \quad b=1,2,
$$

where $\tilde{A}_{\mu}^{b}=\bar{\chi} \gamma_{\mu} \gamma_{5} \frac{\tau^{b}}{2} \chi$ is the axial vector current and $\tilde{P}^{b}=\bar{\chi} \gamma_{5} \frac{\tau^{b}}{2} \chi$ the pseudoscalar density in the twisted basis. The large $t / a$ limit is required in order to isolate the contribution of the lowest-lying charged pseudoscalar meson state in the correlators of Eq. (7). This way of determining $m_{\mathrm{PCAC}}$ is equivalent to imposing on the lattice the validity of the axial Ward identity $\partial_{\mu} \tilde{A}_{\mu}^{b}=2 m_{\mathrm{PCAC}} \tilde{P}^{b}$, $b=1,2$, between the vacuum and the charged zero threemomentum one-pion state. When $m_{0}$ is taken such that $m_{\mathrm{PCAC}}$ vanishes, this Ward identity expresses isospin conservation, as it becomes clear by rewriting it in the physical quark basis. The value of $m_{\mathrm{cr}}$ is determined at each $\beta$ value at the lowest twisted mass, a procedure that preserves $\mathcal{O}(a)$ improvement and keeps $\mathcal{O}\left(a^{2}\right)$ small [31,32].

The twisted mass fermionic action breaks parity and isospin at finite lattice spacing, as it is apparent from the form of the Wilson term in Eq. (6). In particular, the isospin breaking in physical observables is a cutoff effect of $\mathcal{O}\left(a^{2}\right)$ [2]. However the up- and down-propagators satisfy $G_{u}(x, y)=\gamma_{5} G_{d}^{\dagger}(y, x) \gamma_{5}$, which means that two-point correlators are equal with their Hermitian conjugate with $u$ and $d$-quarks interchanged. Using in addition that the masses are computed from real correlators, it leads to the following pairs being degenerate: $\pi^{+}$and $\pi^{-}$, proton and neutron, and $\Delta^{++}\left(\Delta^{+}\right)$and $\Delta^{-}\left(\Delta^{0}\right)$. A theoretical analysis [29] shows that potentially large $\mathcal{O}\left(a^{2}\right)$ effects that appear in the $\pi^{0}$-mass are suppressed in other quantities. Calculation of the mass of $\pi^{0}$, which requires the evaluation of disconnected diagrams, has been carried out confirming large $\mathcal{O}\left(a^{2}\right)$-effects. In the baryon sector we study isospin breaking by evaluating the mass difference between $\Delta^{++}\left(\Delta^{-}\right)$and $\Delta^{+}\left(\Delta^{0}\right)$. Since no disconnected contributions enter, the mass splitting can be evaluated using fixed source propagators. An accurate evaluation of the isospin splitting and its dependence on the lattice spacing is an important component of this work. Examining the size of isospin breaking is a crucial aspect, in particular, regarding future applications of twisted mass fermions to study baryon structure. We find that the isospin breaking for the values of the lattice spacing considered in this work is consistent with zero within our statistical accuracy. Taking the continuum limit of our lattice results we confirm that $\Delta^{++,-}$and $\Delta^{+, 0}$ are indeed degenerate leading to the same mass at the physical point.

\section{LATTICE TECHNIQUES}

The simulation parameters were chosen such that the pion mass ranges from about $300-600 \mathrm{MeV}$. The lattice volumes and masses used in this calculation are collected in Table I. Finite size effects are examined using the smallest pion mass at $\beta=3.9$ as finite volume effects are largest. At this mass we have simulations on lattices of spatial size, $L_{s} \sim 2.1 \mathrm{fm}$ and $L_{s} \sim 2.7 \mathrm{fm}$.

In order to estimate finite $a$-effects and the continuum limit we use two sets of results at $\beta=3.9$ and $\beta=4.05$. Although a further set of gauge configurations at $\beta=3.8$ is analyzed this set is not used to extrapolate to the continuum limit. The reason is that the performance of the HMC algorithm that we use for the simulations deteriorates when we go to small $\mu$ values on this coarser lattice. The long autocorrelation times of the plaquette and the PCAC mass that we observe [13] make the tuning to maximal twist less reliable than for the finer lattices. An analysis aimed at quantifying the impact of possible numerical errors from the tuning procedure on observables [7] is still in progress. Therefore the set at $\beta=3.8$ is used only as a cross-check and to estimate cutoff errors. 
TABLE I. The parameters of our calculation.

\begin{tabular}{|c|c|c|c|c|c|}
\hline $\begin{array}{ll}\beta=4.05, & a=0.0667(5) \mathrm{fm} \\
32^{3} \times 64, & L_{s}=2.1 \mathrm{fm}\end{array}$ & $\begin{array}{c}a \mu \\
m_{\pi}(\mathrm{GeV})\end{array}$ & $\begin{array}{l}0.0030 \\
0.3070(18)\end{array}$ & $\begin{array}{l}0.0060 \\
0.4236(18)\end{array}$ & $\begin{array}{l}0.0080 \\
0.4884(15)\end{array}$ & $\begin{array}{l}0.0120 \\
0.5981(18)\end{array}$ \\
\hline $\begin{array}{l}\beta=3.9, \quad a=0.0855(5) \mathrm{fm} \\
24^{3} \times 48, \quad L_{s}=2.1 \mathrm{fm}\end{array}$ & $\begin{array}{c}a \mu \\
m_{\pi}(\mathrm{GeV})\end{array}$ & $\begin{array}{l}0.0040 \\
0.3131(16)\end{array}$ & $\begin{array}{l}0.0064 \\
0.3903(9)\end{array}$ & $\begin{array}{l}0.0085 \\
0.4470(12)\end{array}$ & $\begin{array}{l}0.010 \\
0.4839(12)\end{array}$ \\
\hline $32^{3} \times 64, \quad L_{s}=2.7 \mathrm{fm}$ & $\begin{array}{c}a \mu \\
m_{\pi}(\mathrm{GeV})\end{array}$ & $\begin{array}{l}0.0040 \\
0.3082(55)\end{array}$ & & & \\
\hline $\begin{array}{l}\beta=3.8, \quad a=0.0995(7) \mathrm{fm} \\
24^{3} \times 48, \quad L_{s}=2.4 \mathrm{fm}\end{array}$ & $\begin{array}{c}a \mu \\
m_{\pi}(\mathrm{GeV})\end{array}$ & $\begin{array}{l}0.0060 \\
0.3667(17)\end{array}$ & $\begin{array}{l}0.0080 \\
0.4128(16)\end{array}$ & $\begin{array}{l}0.0110 \\
0.4799(9)\end{array}$ & $\begin{array}{l}0.0165 \\
0.5855(10)\end{array}$ \\
\hline
\end{tabular}

\section{A. Interpolating fields}

The masses of the nucleon and the $\Delta$ 's are extracted from two-point correlators using the standard interpolating fields, which for the proton, the $\Delta^{++}$and $\Delta^{+}$, are given by

$$
\begin{gathered}
J_{p}=\epsilon_{a b c}\left(u_{a}^{T} C \gamma_{5} d_{b}\right) u_{c}, \quad J_{\Delta^{++}}^{\mu}=\epsilon_{a b c}\left(u_{a}^{T} C \gamma^{\mu} u_{b}\right) u_{c}, \\
J_{\Delta^{+}}^{\mu}=\frac{1}{\sqrt{3}} \epsilon_{a b c}\left[2\left(u_{a}^{T} C \gamma^{\mu} d_{b}\right) u_{c}+\left(u_{a}^{T} C \gamma^{\mu} u_{b}\right) d_{c}\right],
\end{gathered}
$$

where $C=\gamma_{4} \gamma_{2}$.

Local interpolating fields are not optimal for suppressing excited state contributions. We apply Gaussian smearing to each quark field, $q(\mathbf{x}, t)[33,34]$. The smeared quark field is given by $q^{\text {smear }}(\mathbf{x}, t)=\sum_{\mathbf{y}} F(\mathbf{x}, \mathbf{y} ; U(t)) q(\mathbf{y}, t)$ using the gauge invariant smearing function

$$
F(\mathbf{x}, \mathbf{y} ; U(t))=(1+\alpha H)^{n}(\mathbf{x}, \mathbf{y} ; U(t)),
$$

constructed from the hopping matrix understood as a matrix in coordinate, color, and spin space,

$$
\begin{aligned}
H(\mathbf{x}, \mathbf{y} ; U(t))= & \sum_{i=1}^{3}\left(U_{i}(\mathbf{x}, t) \delta_{\mathbf{x}, \mathbf{y}-a \hat{i}}\right. \\
& \left.+U_{i}^{\dagger}(\mathbf{x}-a \hat{i}, t) \delta_{\mathbf{x}, \mathbf{y}+a \hat{i}}\right)
\end{aligned}
$$

The parameters $\alpha$ and $n$ are varied so that the root mean square (r.m.s.) radius obtained using the proton interpolating field is in the range of $0.3-0.4 \mathrm{fm}$. In Fig. 1 we show lines of constant r.m.s. radius as we vary $\alpha$ and $n$. The larger the $n$ the more time consuming is the smearing procedure. On the other hand, for $\alpha \gtrsim 1$, increasing further $\alpha$ does not reduce $n$ significantly. Therefore, we choose a value of $\alpha$ large enough so that the weak $\alpha$-dependence sets in, and we adjust $n$ to obtain the required value of the r.m.s. radius. We consider two sets for these parameters giving r.m.s. radius $0.32 \mathrm{fm}$ and $0.41 \mathrm{fm}$, as shown in Fig. 1. For each set of parameters we evaluate the effective mass as

$$
m_{\text {eff }}^{P}=-\log \left(C_{P}(t) / C_{P}(t-1)\right),
$$

where $C_{P}(t)$ is the zero-momentum two-point correlator of the particle $P$ given by

$$
\begin{aligned}
C_{P}(t) & =\frac{1}{2} \operatorname{Tr}\left(1 \pm \gamma_{4}\right) \sum_{\mathbf{x}_{\text {sink }}}\left\langle J_{P}\left(\mathbf{x}_{\text {sink }}, t_{\text {sink }}\right) \bar{J}_{P}\left(\mathbf{x}_{\text {source }}, t_{\text {source }}\right)\right\rangle, \\
t & =t_{\text {sink }}-t_{\text {source }} .
\end{aligned}
$$

In Fig. 2, we show the nucleon effective mass, $m_{\mathrm{eff}}^{N}$ in lattice units for 10 configurations at $\beta=3.9$ and $a \mu=$ 0.0085 . For the optimization of the parameters we apply Gaussian smearing at the sink, whereas for the source we use local interpolating fields so that no additional inversions are needed when we change $\alpha$ and $n$. As can be seen, for both sets of smearing parameters, the excited state contributions are suppressed with the set $\alpha=4, n=50$ producing a plateau a couple of time slices earlier. If, in addition, we apply APE smearing [35] to the spatial links that enter the hopping matrix in the smearing function, then gauge noise is reduced resulting in a better identification of the plateau. Therefore for all computations at $\beta=3.9$ we use Gaussian smearing with $\alpha=4$ and $n=50$. Having chosen the smearing parameters, for the results that follow, we apply smearing at the source and compute the mass

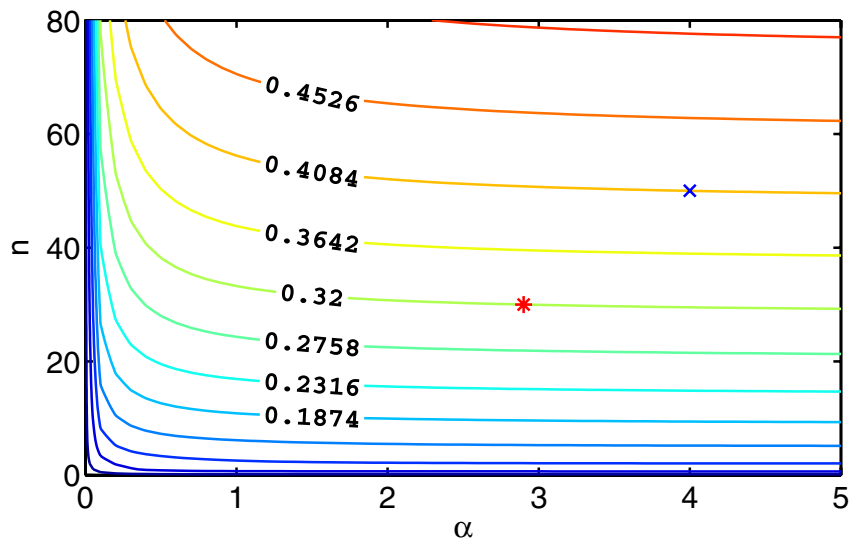

FIG. 1 (color online). Lines of constant r.m.s. radius as a function of the smearing parameters $\alpha$ and $n$. The asterisk shows the values $\alpha=2.9, n=30$ and the cross $\alpha=4.0, n=$ 50 . 


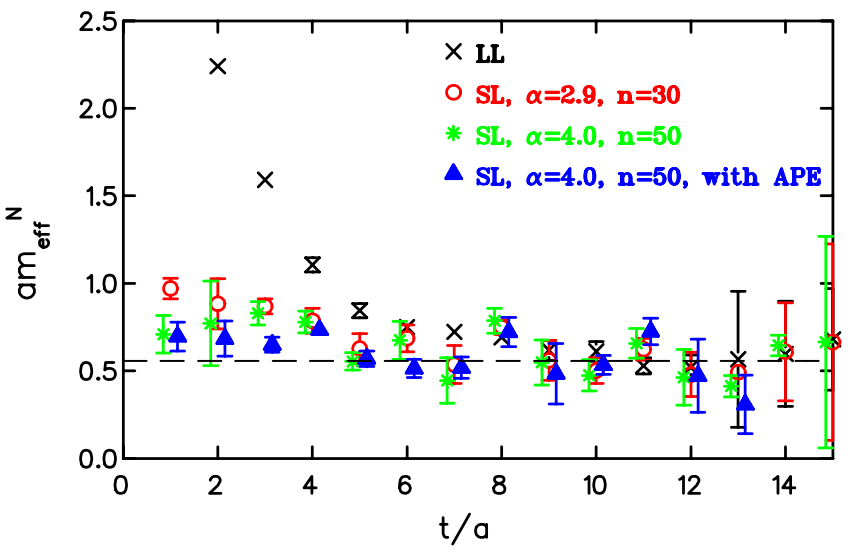

FIG. 2 (color online). $\quad m_{\mathrm{eff}}^{N}$ versus time separation both in lattice units. Crosses show results using local sink and source (LL), circles (asterisks) using Gaussian smearing at the sink (SL) with $\alpha=2.9$ and $n=30 \quad(\alpha=4$ and $n=50)$, and filled triangles with $\alpha=4$ and $n=50$ and APE smearing. The dashed line is the plateau value extracted by fitting results when APE smearing is used.

using both local (LS) and smeared sink (SS). For $\beta=4.05$ we readjust the parameters so that the nucleon r.m.s. radius is still about $0.4 \mathrm{fm}$, obtaining $\alpha=4$ and $n=70$. For $\beta=$ 3.8 we use $\alpha=4$ and 30 to keep the r.m.s. radius at the same value. Also for these two values of $\beta$ we apply APE smearing to the gauge links that are used in $F(\mathbf{x}, \mathbf{y} ; U(t))$.

There are other methods to enhance ground state dominance besides Gaussian smearing. Smearing based on link fuzzing has been effectively used in the pion sector. In this work, having optimized our parameters for Gaussian smearing we use only local and Gaussian-smeared interpolating fields.

\section{B. Two-point correlators}

The lowest energy state with which the nucleon interpolating field given in Eq. (8) has a nonvanishing overlap is the proton state $|p(\mathbf{p}, s)\rangle$

$$
\left\langle 0\left|J_{p}\right| p(\mathbf{p}, s)\right\rangle=Z_{p} u(\mathbf{p}, s) .
$$

$Z_{p}$ is a constant overlap factor and $u(\mathbf{p}, s)$, with $s \in$ $\{-1 / 2,+1 / 2\}$, is a solution of the Dirac equation

$$
\left(\not p-m_{N}\right) u=0 .
$$

Averaging over the nucleon spins and choosing the nucleon rest frame, we are led to the two-point correlator

$$
\begin{aligned}
C_{N}^{ \pm}(t)= & \frac{1}{2} \operatorname{Tr}\left(1 \pm \gamma_{4}\right) \sum_{\mathbf{x}_{\text {sink }}}\left\langle J_{N}\left(\mathbf{x}_{\text {sink }}, t_{\text {sink }}\right)\right. \\
& \left.\times \bar{J}_{N}\left(\mathbf{x}_{\text {source }}, t_{\text {source }}\right)\right\rangle \\
t= & t_{\text {sink }}-t_{\text {source }} .
\end{aligned}
$$

Space-time reflection symmetries of the action and the antiperiodic boundary conditions in the temporal direction for the quark fields imply, for zero three-momentum correlators, that $C_{N}^{+}(t)=-C_{N}^{-}(T-t)$. The nucleon mass is extracted from the exponential decay of the correlator

$$
C_{N}(t)=C_{N}^{+}(t)-C_{N}^{-}(T-t) .
$$

To increase the precision we also average over the proton and neutron correlators since these are degenerate in mass.

In analogy to Eq. (13), the overlap of the $\Delta^{+}$interpolating field with the $\Delta^{+}$state is given by

$$
\left\langle 0\left|J_{\Delta^{+}}^{\mu}\right| \Delta^{+}(\mathbf{p}, s)\right\rangle=Z_{\Delta^{+}} u^{\mu}(\mathbf{p}, s) .
$$

Every vector component of the Rarita-Schwinger spinor $u^{\mu}$ satisfies the Dirac equation

$$
\left(\not p-m_{\Delta}\right) u^{\mu}=0, \quad \mu=1 \ldots 4,
$$

and in addition the auxiliary conditions

$$
p_{\mu} u^{\mu}=0 \text { and } \gamma_{\mu} u^{\mu}=0
$$

are fulfilled. The four independent solutions are labeled by $s \in\{-3 / 2,-1 / 2,1 / 2,3 / 2\}$. The $\Delta$ interpolating fields as defined in Eq. (8) have overlap also with the heavier spin- $1 / 2$ excitations. These overlaps can be removed when the conditions in Eq. (19) are enforced on the interpolating fields. This can be achieved by the incorporation of a spin-3/2 projector in the definitions of the interpolating fields

$$
\begin{gathered}
J_{3 / 2}^{\mu}=P_{3 / 2}^{\mu \nu} J_{\nu \Delta}, \\
P_{3 / 2}^{\mu \nu}=\delta^{\mu \nu}-\frac{1}{3} \gamma^{\mu} \gamma^{\nu}-\frac{1}{3 p^{2}}\left(\not p \gamma^{\mu} p^{\nu}+p^{\mu} \gamma^{\nu} \not p\right)
\end{gathered}
$$

Similarly the spin-1/2-interpolating field, $J_{1 / 2}^{\mu}$, that has only overlap with the $1 / 2$ state, is obtained by acting with the spin- $1 / 2$ projector $P_{1 / 2}^{\mu \nu}=g^{\mu \nu}-P_{3 / 2}^{\mu \nu}$ on $J_{\Delta}^{\mu}$. Using any of the three interpolating fields, the $\Delta$ masses are extracted from the two-point functions

$$
\begin{aligned}
C_{\Delta}^{ \pm}(t)= & \frac{1}{6} \operatorname{Tr}\left(1 \pm \gamma_{4}\right) \sum_{\mathbf{x}_{\text {sink }}} \sum_{i=1}^{3}\left\langle J_{\Delta}^{i}\left(\mathbf{x}_{\text {sink }}, t_{\text {sink }}\right)\right. \\
& \left.\times \bar{J}_{\Delta}^{i}\left(\mathbf{x}_{\text {source }}, t_{\text {source }}\right)\right\rangle, \\
t= & t_{\text {sink }}-t_{\text {source }}
\end{aligned}
$$

Figure 3 compares effective masses extracted from correlation functions with and without the spin-3/2 projection at $\beta=3$.9. For this comparison we use 90 configurations, a number sufficient for the purpose of this check. The results for the effective mass are hardly affected by including the spin-3/2 projector even at very short time separations. This is because the overlap of the interpolating field $J_{\Delta}^{\mu}$ with the spin- $1 / 2$ state is small, a property that holds at all values of $\beta$. This is clearly seen in Fig. 4 at $\beta=3.8$ where the effective mass obtained using the spin- $1 / 2$ projected interpolating field $J_{1 / 2}^{\mu}$ is much more noisy than with $J_{3 / 2}^{\mu}$ due to 


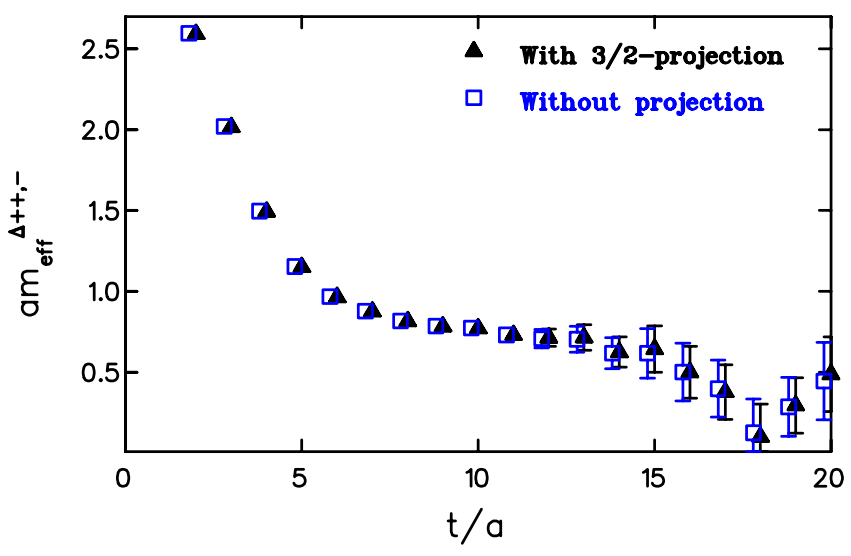

FIG. 3 (color online). Comparison of effective masses for $\Delta^{++,-}$for $a \mu=0.0085$ at $\beta=3.9$ on the lattice volume $24^{3} \times 48$, obtained with (filled triangles) or without (open squares shifted to the left for clarity) spin projection, using a sample of 90 configurations. The mass difference with projection and without projection is much smaller than the statistical error.

the small overlap with the spin- $1 / 2$ state. This behavior is in agreement with the results of Ref. [36] where the same spin projections were implemented. Since the impact on the plateau value is negligible compared to the statistical uncertainty, we use only the nonprojected interpolating fields from here on. We average the correlators of $\Delta^{++}$ with $\Delta^{-}$as well as $\Delta^{+}$with $\Delta^{0}$. We do not average the $\Delta^{++}$and $\Delta^{+}$correlators as they differ by an $O\left(a^{2}\right)$ isospin breaking effect.

\section{Effective masses}

In Fig. 5 we show the nucleon effective masses at $\beta=$ 3.9 on a volume $24^{3} \times 48$ for all the values of $\mu$ considered. We smeared the source as described in the previous section and either use a local sink or smear the sink with the same smearing used for the source. As expected, the

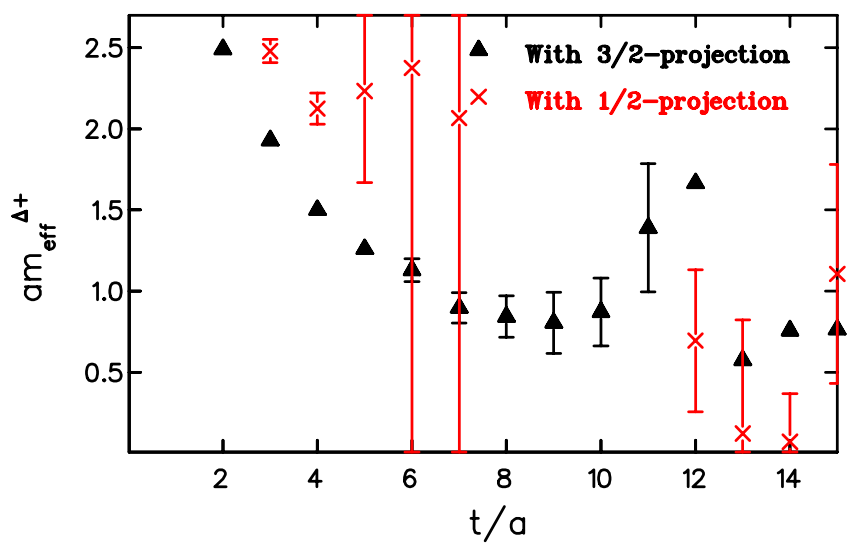

FIG. 4 (color online). Comparison of effective masses for $\Delta^{+}$ for $a \mu=0.011$ at $\beta=3.8$ on the lattice volume $24^{3} \times 48$, obtained with 3/2-spin (filled triangles) or with 1/2-spin projection, using a sample of 50 configurations.

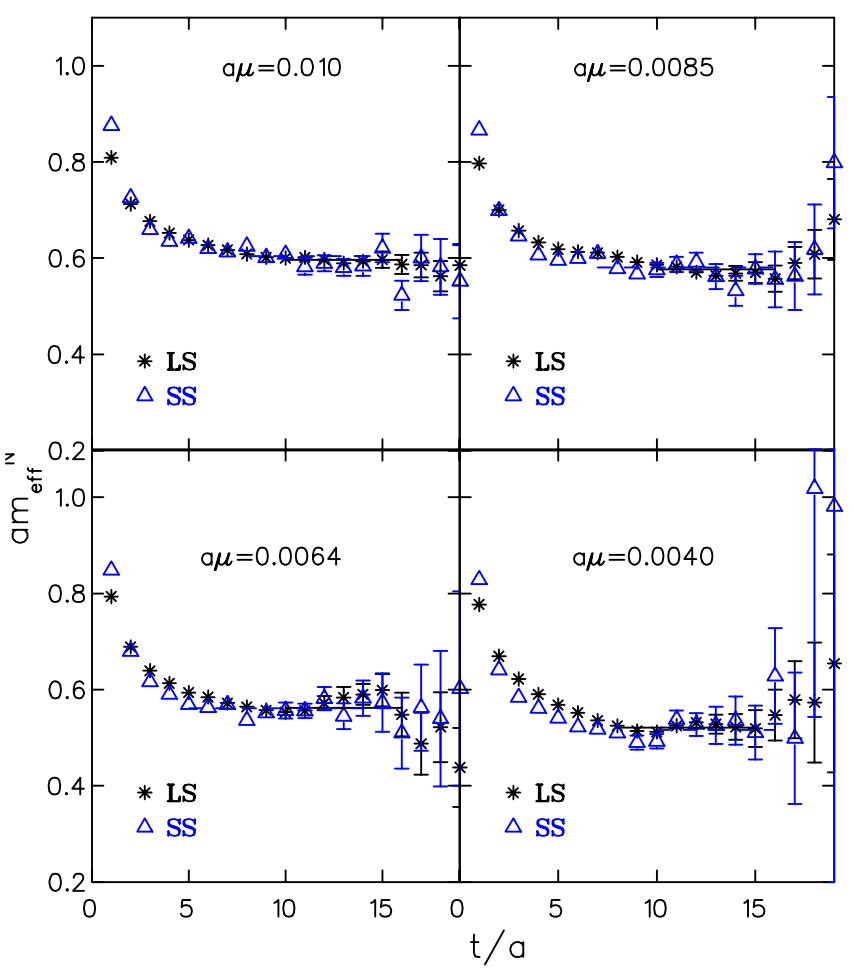

FIG. 5 (color online). Nucleon effective mass (LS: asterisks, SS: open triangles) for $\beta=3.9$ versus time separation in lattice units, for $a \mu=0.010$ (upper left), 0.0085 (upper right), 0.0064 (lower left), and 0.0040 (lower right). The constant lines are the best fits to the data over the range spanned by the lines.

effective masses are consistent for both smeared and local sink yielding asymptotically the same constant. We fit the effective mass to a constant in the region where $m_{\text {eff }}(t)$ becomes time independent (plateau region) and vary the lower $t$-range of the fit so that $\chi^{2}$ per degree of freedom (d.o.f.) becomes less than one. We take this value for the mass of the nucleon. In Fig. 6 we show, for the same $\mu$-values, effective masses for the $\Delta^{++,-}$and $\Delta^{+, 0}$ using smeared source and sink. We fit in the same way as in the nucleon case to extract the mass of the $\Delta$. As can be seen, the quality of the plateaus in the nucleon case is better than in the case of the $\Delta$. This explains why results on the $\Delta$ mass have larger errors. The errors are evaluated using jackknife and the $\Gamma$-method [37] to check consistency. The integrated autocorrelation times for our baryonic observables are very small for our configuration ensembles. Since for our computation we use gauge configurations that are separated by $8-20$ trajectory lengths, autocorrelations are negligible.

The resulting masses using local and smeared interpolating fields are summarized in Table II for $\beta=3.9$ using lattice sizes of $24^{3} \times 48$ and $32^{3} \times 64$, while those obtained for $\beta=4.05$ on a lattice volume of $32^{3} \times 64$ are reported in Table III. Results obtained at $\beta=3.8$ are given in Table IV. The mass of the pion listed in Table II is taken from Ref. [13] and was evaluated using a larger set of 


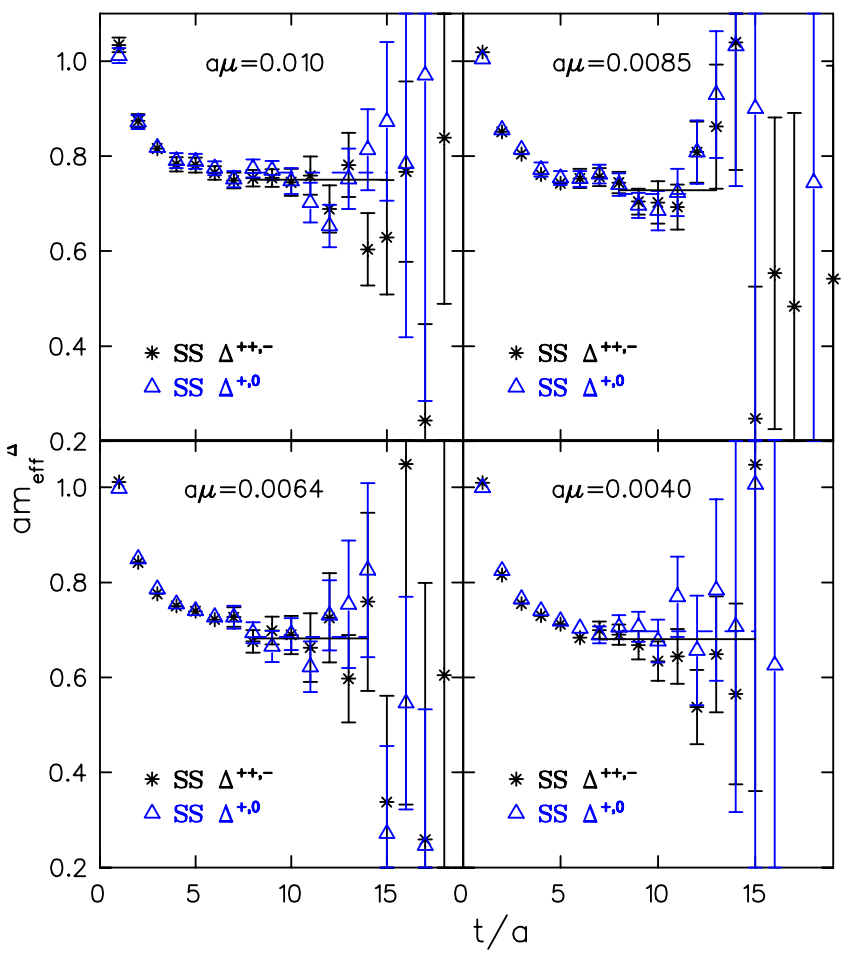

FIG. 6 (color online). $\Delta^{++,-}$(asterisks) and $\Delta^{+, 0}$ (open triangles) effective masses using smeared-smeared (SS) correlators for $\beta=3.9$ versus time separation in lattice units, for $a \mu=0.010$ (upper left), 0.0085 (upper right), 0.0064 (lower left), and 0.0040 (lower right). The straight lines are the best fits to the data over the range spanned by the lines. The solid line is for $\Delta^{++,-}$and the dashed for $\Delta^{+, 0}$ and they coincide. configurations applying a different smearing than the one used in this work. A detailed description of this evaluation as well as the error analysis is presented in Ref. [31]. The pion masses given in Tables III and IV are again obtained in a separate evaluation [38].

\section{LATTICE ARTIFACTS}

\section{A. Finite volume effects}

At $\beta=3.9$ and for $a \mu=0.004$ we have gauge configurations on two lattices of different volume. This is the smallest $\mu$-value considered at $\beta=3.9$ and it is the one that potentially can have the largest finite volume effects. On the lattice of spatial extension $L_{s}=24$ the other three larger $\mu$-values satisfy the condition $m_{\pi} L_{s} \geq 4$, whereas for $a \mu=0.004$ we have $m_{\pi} L_{s} \sim 3.2$. On the $32^{3}$ lattice at $a \mu=0.004$ we have $m_{\pi} L_{s}>4$. Applying the resummed Lüscher formula to the nucleon mass and using the knowledge of the $\pi N$ scattering amplitude to $\mathcal{O}\left(p^{2}\right)$ and $\mathcal{O}\left(p^{4}\right)$, the volume corrections are estimated to be about $3 \%$ to $5 \%$ [39] for $L_{s} \sim 2 \mathrm{fm}$ and $m_{\pi} \sim 300 \mathrm{MeV}$. In Table II we give the results for the nucleon mass using our two lattice volumes. The smaller lattice volume has spatial length very close to the $2 \mathrm{fm}$ length of Ref. [39] namely $L_{s} \sim 2.1 \mathrm{fm}$. The results for $m_{N}$ do not change within our statistical accuracy when we use the larger lattice size of $L_{s} \sim$ $2.7 \mathrm{fm}$. We make the assumption that for the larger lattice finite volume corrections have become negligible and take them to be a good approximation to the infinite volume results. In other words we take $m_{N}\left(L_{s}=\infty\right) \simeq m_{N}\left(L_{s}=\right.$ $2.7 \mathrm{fm})$. This assumption was shown to be valid in the pion sector where a finite size analysis was carried out [31]. We

TABLE II. Results for the nucleon and $\Delta$ mass at $\beta=3.9$ for lattices of size $24^{3} \times 48$ and $32^{3} \times 64$. LL stands for local sink and local source, LS for local sink and smeared source, and SS for smeared sink and smeared source. The results for the pion mass are taken from Table 2 of Ref. [13] computed using more gauge configurations than we used for the evaluation of the baryon masses as well as a different smearing and therefore are the same for LL, LS, and SS. With an asterisk we mark results for which the effective mass does not show a good plateau.

\begin{tabular}{|c|c|c|c|c|c|c|}
\hline$a \mu$ & Interpolating field & Number of confs. & $a m_{\pi}$ & $a m_{N}$ & $a m_{\Delta^{++,-}}$ & $a m_{\Delta^{+, 0}}$ \\
\hline \multicolumn{7}{|c|}{$24^{3} \times 48$} \\
\hline 0.0040 & LL & 471 & $0.13587(68)$ & $0.511(11)$ & $0.699(15)$ & $0.708(25)$ \\
\hline 0.0040 & LS & 419 & $0.13587(68)$ & $0.521(6)$ & $0.694(11)$ & $0.717(13)$ \\
\hline 0.0040 & SS & 419 & $0.13587(68)$ & $0.515(5)$ & $0.682(12)$ & $0.697(17)$ \\
\hline 0.0064 & LL & 199 & $0.16937(36)$ & $0.565(11)$ & $0.727(15)$ & $0.763(15)$ \\
\hline 0.0064 & LS & 235 & $0.16937(36)$ & $0.565(6)$ & $0.715(13)$ & $0.742(7)$ \\
\hline 0.0064 & SS & 235 & $0.16937(36)$ & $0.561(4)$ & $0.710(11)$ & $0.711(10)$ \\
\hline 0.0085 & LL & 153 & $0.19403(50)$ & $0.568(8)$ & $0.754(12)$ & $0.776(22)$ \\
\hline 0.0085 & LS & 186 & $0.19403(50)$ & $0.581(6)$ & $0.746(9)$ & $0.751(12)$ \\
\hline 0.0085 & SS & 186 & $0.19403(50)$ & $0.580(6)$ & $0.738(11)$ & $0.742(9)$ \\
\hline 0.0100 & LL & 173 & $0.21004(52)$ & $0.613(6)$ & $0.823(7)^{*}$ & $0.767(12)$ \\
\hline 0.0100 & $\mathrm{LS}$ & 213 & $0.21004(52)$ & $0.595(7)$ & $0.742(7)$ & $0.760(7)$ \\
\hline 0.0100 & SS & 213 & $0.21004(52)$ & $0.589(9)$ & $0.750(10)$ & $0.755(10)$ \\
\hline \multicolumn{7}{|c|}{$32^{3} \times 64$} \\
\hline 0.0040 & LS & 201 & $0.13377(24)$ & $0.518(5)$ & $0.672(9)$ & $0.670(14)$ \\
\hline 0.0040 & SS & 201 & $0.13377(24)$ & $0.510(5)$ & $0.660(9)$ & $0.660(14)$ \\
\hline
\end{tabular}


TABLE III. Results for the nucleon and $\Delta$ mass at $\beta=4.05$ for the $32^{3} \times 64$ lattice. LL stands for local sink and local source, LS for local sink and smeared source, and SS for smeared sink and smeared source. The results for the pion mass are computed using more gauge configurations than we used for the evaluation of the baryon masses as well as a different smearing [38] and therefore are the same for LL, LS, and SS. With an asterisk we mark results for which the effective mass does not show a good plateau. Empty entries are due to the absence of a sufficient plateau region.

\begin{tabular}{|c|c|c|c|c|c|c|}
\hline$a \mu$ & Interpolating field & Number of confs. & $a m_{\pi}$ & $a m_{N}$ & $a m_{\Delta^{++,-}}$ & $a m_{\Delta^{+, 0}}$ \\
\hline 0.0030 & LL & 70 & $0.1038(6)$ & $0.403(15)$ & $0.633(30)$ & \\
\hline 0.0030 & LS & 201 & $0.1038(6)$ & $0.396(7)$ & $0.536(18)$ & $0.546(12)$ \\
\hline 0.0030 & SS & 201 & $0.1038(6)$ & $0.402(8)$ & $0.538(19)$ & $0.536(15)$ \\
\hline 0.0060 & LL & 216 & $0.1432(6)$ & $0.453(5)$ & $0.597(8)$ & $0.575(9)$ \\
\hline 0.0060 & LS & 160 & $0.1432(6)$ & $0.448(5)$ & $0.564(7)$ & $0.566(7)$ \\
\hline 0.0060 & SS & 160 & $0.1432(6)$ & $0.446(6)$ & $0.562(6)$ & $0.566(7)$ \\
\hline 0.0080 & LL & 240 & $0.1651(5)$ & $0.465(6)$ & $0.627(6)$ & $0.637(7)$ \\
\hline 0.0080 & LS & 256 & $0.1651(5)$ & $0.469(4)$ & $0.590(7)$ & $0.585(9)$ \\
\hline 0.0080 & SS & 256 & $0.1651(5)$ & $0.465(5)$ & $0.594(7)$ & $0.594(8)$ \\
\hline 0.0120 & LL & 157 & $0.2025(6)$ & $0.520(5)$ & $0.670(4)^{*}$ & $0.677(5)$ \\
\hline 0.0120 & LS & 162 & $0.2025(6)$ & $0.509(4)$ & $0.616(7)^{*}$ & $0.623(7)$ \\
\hline 0.0120 & SS & 162 & $0.2025(6)$ & $0.515(3)$ & $0.616(7)^{*}$ & $0.620(7)$ \\
\hline
\end{tabular}

TABLE IV. Results for the nucleon and $\Delta$ mass at $\beta=3.8$ for the $24^{3} \times 48$ lattice. The notation is the same as that of Table III with SL being a smeared sink and local source.

\begin{tabular}{|c|c|c|c|c|c|c|}
\hline$a \mu$ & Interpolating field & Number of confs. & $a m_{\pi}$ & $a m_{N}$ & $a m_{\Delta^{++,-}}$ & $a m_{\Delta^{+, 0}}$ \\
\hline 0.0060 & LL & 211 & $0.1852(10)$ & $0.623(20)$ & $0.792(25)$ & $0.815(28)$ \\
\hline 0.0060 & SL & 211 & $0.1852(10)$ & $0.637(9)$ & $0.818(11)$ & $0.824(13)$ \\
\hline 0.0080 & LL & 283 & $0.2085(8)$ & $0.676(11)$ & $0.859(11)$ & $0.847(30)$ \\
\hline 0.0080 & SL & 283 & $0.2085(8)$ & $0.665(9)$ & $0.827(17)$ & $0.856(24)$ \\
\hline 0.0110 & LL & 251 & $0.2424(5)$ & $0.700(9)$ & $0.861(13)$ & $0.893(22)$ \\
\hline 0.0110 & SL & 251 & $0.2424(5)$ & $0.699(8)$ & $0.854(14)$ & $0.875(16)$ \\
\hline 0.0165 & LL & 249 & $0.2957(5)$ & $0.759(7)$ & $0.948(12)$ & $0.886(25)$ \\
\hline 0.0165 & SL & 249 & $0.2957(5)$ & $0.744(8)$ & $0.942(13)$ & $0.946(12)$ \\
\hline
\end{tabular}

define the ratio $R_{N} \equiv \Delta m_{N} / m_{N}\left(L_{s}=\infty\right)$, where $\Delta m_{N} \equiv$ $m_{N}\left(L_{s}=2.1 \mathrm{fm}\right)-m_{N}\left(L_{s}=\infty\right)$ and estimate $R_{N}$ with results obtained on our two volumes for the smallest pion mass. This gives us an estimate for our finite volume errors. Using the results tabulated in Table II at $a \mu=0.004$ we conclude that $\Delta m_{N}$ is positive as expected. This is also true for the corresponding difference for the masses of $\Delta^{++,-}$ and $\Delta^{+, 0}$. In Table $\mathrm{V}$ we give the ratios $R_{N}, R_{\Delta^{++,-}}$, and $R_{\Delta^{+, 0}}$. For the nucleon this ratio is compatible with zero and within our accuracy it can be at the most $2 \%$. For the $\Delta^{++,-}$where the statistical errors are smaller than for the $\Delta^{+, 0}$, the volume corrections range from $1 \%$ to $5 \%$. From this study we conclude that finite volume effects on the nucleon mass are negligible whereas for the $\Delta$ we can at most have corrections on the few percent level.

\section{B. Isospin breaking}

One of the main goals of this work is to examine isospin breaking in the baryon sector due to lattice artifacts. As already explained the proton and the neutron are degenerate. Isospin breaking in the light baryon sector can be

TABLE V. Finite volume dependence at $\beta=3.9$ for $a \mu=0.004$. Results with a lattice of size $24^{3} \times 48$ are compared to those obtained with a lattice size of $32^{3} \times 64$. For a hadron state $P$ we define $R_{P} \equiv\left(m_{P}\left(L_{s}=2.1 \mathrm{fm}\right)-m_{P}\left(L_{s}=2.7 \mathrm{fm}\right)\right) / m_{P}\left(L_{s}=2.7 \mathrm{fm}\right) \simeq\left(m_{P}\left(L_{s}=\right.\right.$ $\left.2.1 \mathrm{fm})-m_{P}(\infty)\right) / m_{P}(\infty)$ assuming the masses at $2.7 \mathrm{fm}$ to be close enough to the infinite volume limit.

\begin{tabular}{lcccc}
\hline \hline$a \mu$ & Interpolating field & $R_{N}$ & $R_{\Delta^{++,-}}$ & $R_{\Delta^{+, 0}}$ \\
\hline 0.0040 & LS & $0.006(15)$ & $0.033(22)$ & $0.070(26)$ \\
0.0040 & SS & $0.010(14)$ & $0.033(23)$ & $0.056(33)$ \\
\hline \hline
\end{tabular}




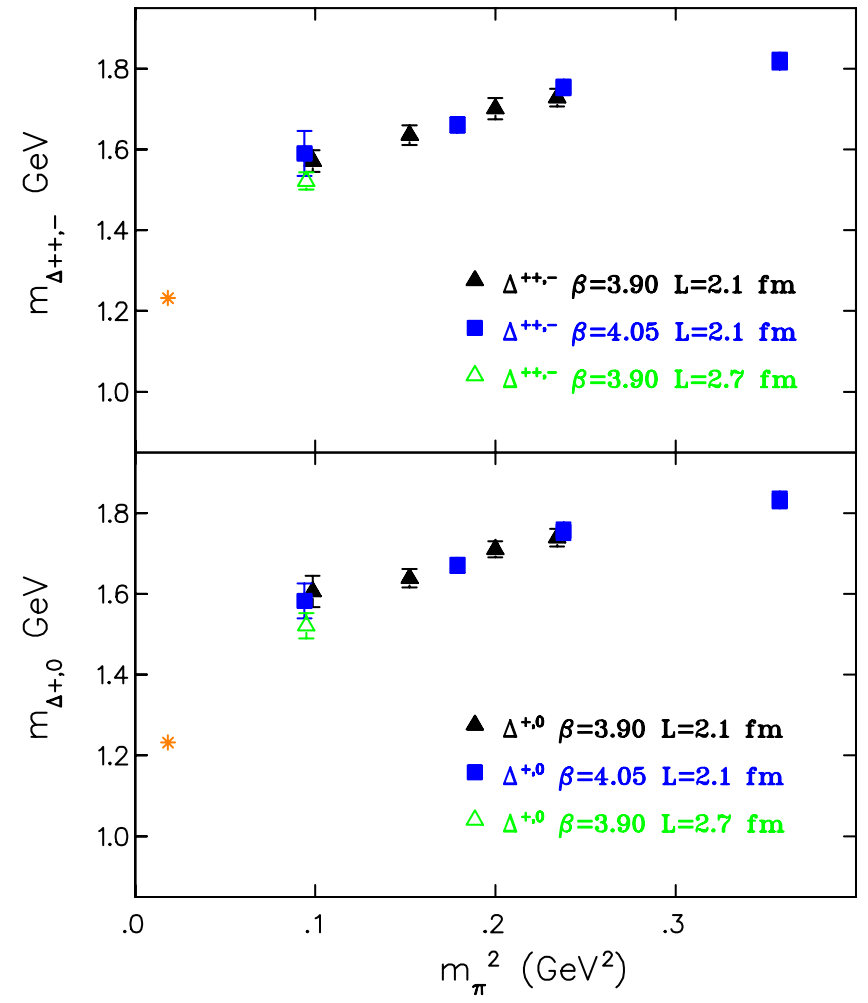

FIG. 7 (color online). The $\Delta^{++,-}$(upper graph) and $\Delta^{+, 0}$ (lower graph) mass as a function of $m_{\pi}^{2}$ for $\beta=3.9$ on a lattice of size $24^{3} \times 48$ (filled triangles) and on a lattice of size $32^{3} \times$ 64 (open triangles). Results at $\beta=4.05$ are shown with the filled squares. The physical $\Delta$ mass is shown with the asterisk.

examined for the $\Delta$. In Fig. 7 we show results for the mass of $\Delta^{++,-}$as well as for the mass of $\Delta^{+, 0}$. Results at $\beta=$ 3.9 and $\beta=4.05$ fall on the same curve pointing to small cutoff effects. Small finite volume effects are visible at the smallest pion mass at $\beta=3.9$ as discussed in the previous

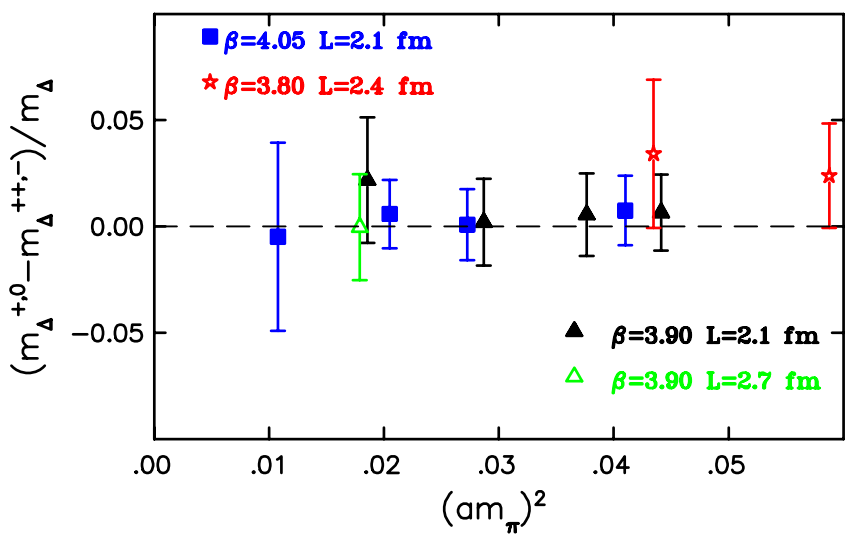

FIG. 8 (color online). The mass splitting between $\Delta^{+, 0}$ and $\Delta^{++,-}$normalized with the mean value of their mass $m_{\Delta}$ as a function of $m_{\pi}^{2}$ in lattice units. Results at $\beta=3.8$ are shown with the asterisks. The rest of the notation is the same as in Fig. 7. subsection. To check for isospin breaking we plot the mass difference between the pairs $\Delta^{++}, \Delta^{-}$and $\Delta^{+}, \Delta^{0}$ normalized by the mean value of their mass in Fig. 8 for $\beta=$ $3.8, \beta=3.9$, and $\beta=4.05$. As can be seen, the splitting is consistent with zero for these values of $\beta$, indicating that isospin breaking in the $\Delta$ system is small.

\section{CHIRAL EXTRAPOLATION}

\section{A. Nucleon mass}

We show our results for the nucleon mass as a function of $m_{\pi}^{2}$ in Fig. 9. The masses are extracted in lattice units. To convert to physical units we need to know the value of the lattice spacing. A standard procedure is to match the experimental value of $f_{\pi}$ to the one obtained on the lattice extrapolated to the physical pion mass. This gives $a=$ $0.0855 \mathrm{fm}$ at $\beta=3.9$ and $a=0.0667 \mathrm{fm}$ at $\beta=4.05$ [14]. We use these values to convert lattice results to physical units. The results at these two $\beta$-values fall on a common curve indicating that cutoff effects are small for these values of the lattice spacing. In Fig. 9 we include, for comparison, results obtained with dynamical staggered fermions from Refs. [40,41]. Results using these two formulations are consistent with each other. As we already discussed, results obtained on lattices of spatial length $L_{s}=2.1 \mathrm{fm}$ and $L_{s}=2.7 \mathrm{fm}$ at $\beta=3.9$ for the lowest pion mass are consistent indicating that finite volume effects are small for the pion masses used in this work. Therefore as a first analysis of our lattice results, we use continuum chiral perturbation theory in an infinite volume to perform the chiral extrapolation to the physical point. An analysis carried out after taking the continuum limit will serve as a check of cutoff effects. The leading one-loop

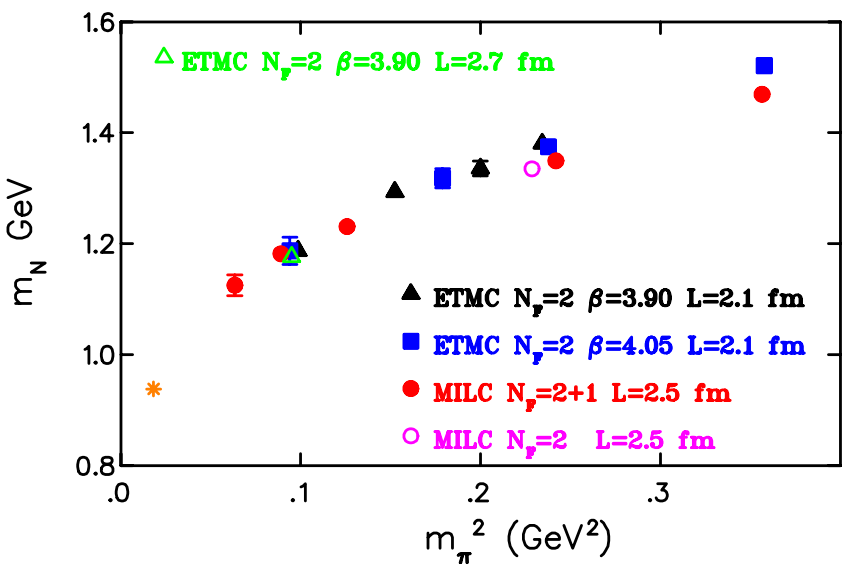

FIG. 9 (color online). The nucleon mass as a function of $m_{\pi}^{2}$ for $\beta=3.9$ on a lattice of size $24^{3} \times 48$ (filled triangles) and on a lattice of size $32^{3} \times 64$ (open triangle). Results at $\beta=$ 4.05 are shown with the filled squares. The physical nucleon mass is shown with the asterisk. Results with dynamical staggered fermions for $N_{F}=2+1$ (filled circles) and $N_{F}=2$ (open circle) on a lattice of size $20^{3} \times 64$ with $a=0.125 \mathrm{fm}$ are from Refs. [40,41]. 
result in heavy baryon chiral perturbation theory $(\mathrm{HB} \chi \mathrm{PT})$ [42] is well known:

$$
m_{N}=m_{N}^{0}-4 c_{1} m_{\pi}^{2}-\frac{3 g_{A}^{2}}{32 \pi f_{\pi}^{2}} m_{\pi}^{3}
$$

with $m_{N}^{0}$, the nucleon mass in the chiral limit, and $c_{1}$ treated as fit parameters. This lowest order result is the same in $\mathrm{HB} \chi \mathrm{PT}$ with dimensional and infrared regularization as well as when the $\Delta$ degree of freedom is explicitly included. It is also the same in manifestly Lorenz-invariant formulation with infrared regularization. Therefore we will use this well-established result to predict the nucleon mass at the physical point as well as fix the lattice spacing using the experimental nucleon mass as input. The higher order results will only be used to estimate the systematic error associated with the chiral extrapolation. We take for $f_{\pi}$ and $g_{A}$ their physical values, namely $f_{\pi}=0.092419(7) \times$ (25) $\mathrm{GeV}$ and $g_{A}=1.2695$ (29), which is what is customarily done in chiral fits to lattice data on the nucleon mass [43-45]. We will take the experimental values for $f_{\pi}$ and $g_{A}$ also when using higher order results. In higher orders new low energy constants enter, and we also fix their values from experimental data. In order to determine the errors on the fit parameters we allow for a variation in the parameters that increases the minimal value of $\chi^{2}$ by one. In Fig. 10 we show the boundary of the allowed variation of the parameters. As expected when the number of available lattice results increases the error decreases. In Fig. 11 we show fits to the $\mathcal{O}\left(p^{3}\right)$ result with the error band determined by the maximum allowed variation in the parameters that increase the minimal $\chi^{2}$ by one. As can be seen, this $\mathcal{O}\left(p^{3}\right)$ result provides a very good fit to our lattice data both at $\beta=3.9$ and $\beta=4.05$. Since finite volume effects are small we use in the fit data on both volumes at $\beta=3.9$.

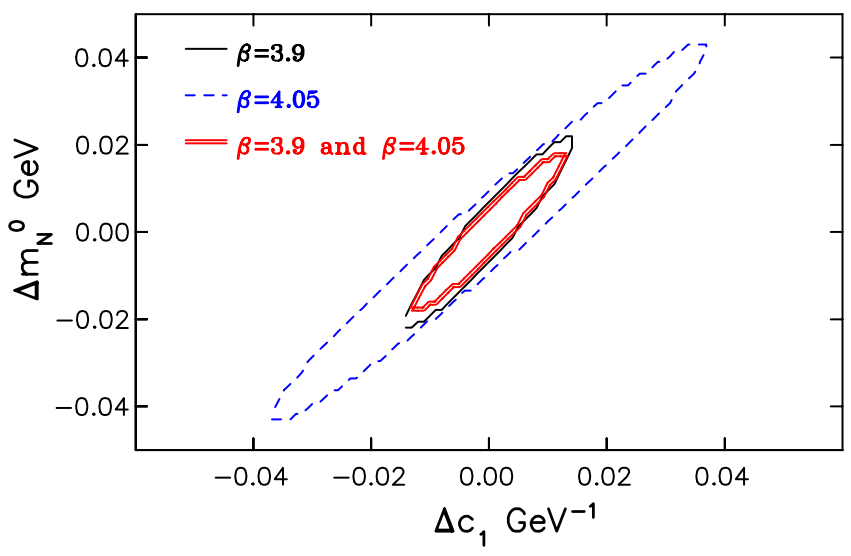

FIG. 10 (color online). The variation of the fit parameters $m_{N}^{0}$ and $c_{1}$. The elliptical boundary is determined by changing these parameters so that the minimal value of $\chi^{2}$ changes by one. The most elongated ellipse is for $\beta=4.05$ using the nucleon mass at the three lighter pion masses, the intermediate is for $\beta=3.9$ using all five points and the smallest is for a combined fit to both $\beta$-values using a total of eight points.

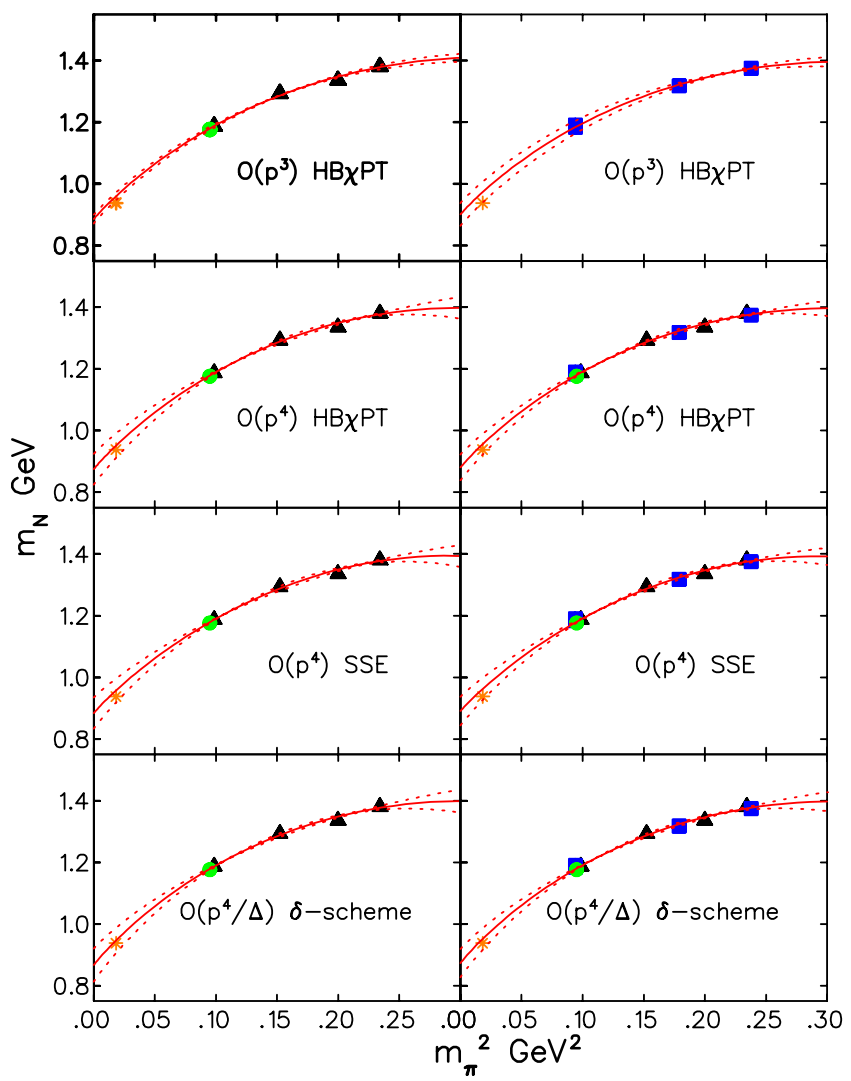

FIG. 11 (color online). On the left set of graphs we show chiral fits to the nucleon mass for $\beta=3.9$ using $a_{\beta=3.9}=0.0855 \mathrm{fm}$ to convert to physical units. On the right set of graphs we show the corresponding chiral fits for $\beta=4.05$ using $a_{\beta=4.05}=$ $0.0667 \mathrm{fm}$. The uppermost graph shows the fit to $\mathcal{O}\left(p^{3}\right)$ $\mathrm{HB} \chi \mathrm{PT}$ where we use our results at the three lowest values of the pion mass. For the higher order fits we perform a simultaneous fit to both $\beta=3.9$ and $\beta=4.05$ always excluding at $\beta=4.05$ the result at the largest pion mass. The physical point, shown by the asterisk, is not included in the fits.

In Table VI we give the values of the parameters $m_{N}^{0}$ and $c_{1}$. In this determination we use the lattice spacing determined from $f_{\pi}$. In the case of $\beta=3.9$ we include the result obtained using the larger volume. We would like to stress that, despite the fact that the physical point is not included in the fit as customarily done in other chiral extrapolations of lattice data. The value of the nucleon mass that we find at the physical pion mass using data at both $\beta=3.9$ and $\beta=4.05$ to fit to the $\mathcal{O}\left(p^{3}\right) \mathrm{HB} \chi \mathrm{PT}$ of Eq. (23) is $963(12) \mathrm{MeV}$, where the error is only statistical.

The nucleon sigma term is defined by

$$
\sigma_{N}=\sum_{q=u, d} \mu_{q} \frac{d M_{N}}{d \mu_{q}}
$$

where we have neglected contributions from other quarks. Following Ref. [46] we use the relation $m_{\pi}^{2} \sim \mu$ to evaluate $\sigma_{N}$ by computing $m_{\pi}^{2} \frac{d M_{N}}{d m_{\pi}^{2}}$. Using the value of $c_{1}$ 
TABLE VI. Fit parameters $m_{N}^{0}$ and $m_{\Delta}^{0}$ in $\mathrm{GeV}$ and $c_{1}$ in $\mathrm{GeV}^{-1}$. Results with an asterisk have $\chi^{2} /$ d.o.f. larger than 1 . All fits to the continuum results excluded the largest value of $r_{0} m_{\pi}$ with the exception of the $\Delta^{+, 0}$ in the $\delta$-scheme where to obtain a good fit we use all six points. For $\beta=3.9$ we use all masses including the results at the larger volume whereas for $\beta=4.05$ we use results at the three smaller pion masses. For the fits to continuum results we give two sets of results: the first set is obtained when using linear interpolation to the reference pion masses and the second using $\mathcal{O}\left(p^{3}\right) \mathrm{HB} \chi \mathrm{PT}$ for interpolation.

\begin{tabular}{|c|c|c|c|c|c|c|c|c|c|c|}
\hline & \multirow{2}{*}{\multicolumn{2}{|c|}{$\beta=3.9$}} & \multirow{2}{*}{\multicolumn{2}{|c|}{$\beta=4.05$}} & \multirow{3}{*}{$\begin{array}{c}\beta=3.9 \text { and } \\
m_{N}^{0}\end{array}$} & \multirow{3}{*}{$\begin{array}{c}\beta=4.05 \\
c_{1}\end{array}$} & \multicolumn{2}{|c|}{ Continuum linear } & \multicolumn{2}{|c|}{ Continuum with } \\
\hline & & & & & & & & & $\mathcal{O}\left(p^{3}\right)$ & $\mathrm{HB} \chi \mathrm{PT}$ \\
\hline & $m_{N}^{0}$ & $c_{1}$ & $m_{N}^{0}$ & $c_{1}$ & & & $m_{N}^{0}$ & $c_{1}$ & $m_{N}^{0}$ & $c_{1}$ \\
\hline \multicolumn{11}{|l|}{ Nucleon } \\
\hline $\mathcal{O}\left(p^{3}\right) \mathrm{HB} \chi \mathrm{PT}$ & $0.886(14)$ & $-1.21(2)$ & $0.901(37)$ & $-1.18(4)$ & $0.889(13)$ & $-1.20(2)$ & $0.904(14)$ & $-1.19(2)$ & $0.898(9)$ & $-1.19(1)$ \\
\hline $\mathcal{O}\left(p^{4}\right) \quad \mathrm{HB} \chi \mathrm{PT}$ & $0.875(50)$ & $-1.23(17)$ & 0.929 & -1.10 & $0.881(42)$ & $-1.22(12)$ & $0.893(47)$ & $-1.21(12)$ & $0.889(25)$ & $-1.21(7)$ \\
\hline $\mathcal{O}\left(p^{4}\right) \mathrm{SSE}$ & $0.884(51)$ & $-1.19(14)$ & 0.944 & -1.02 & $0.891(47)$ & $-1.17(15)$ & $0.903(52)$ & $-1.16(15)$ & $0.901(30)$ & $-1.15(9)$ \\
\hline $\mathcal{O}\left(p^{4} / \Delta\right) \delta$-scheme & $0.867(54)$ & $-1.29(18)$ & 0.927 & -1.13 & $0.873(46)$ & $-1.28(15)$ & $0.886(51)$ & $-1.27(16)$ & $0.883(29)$ & $-1.26(9)$ \\
\hline$\Delta^{++,-}$ & $m_{\Delta}^{0}$ & $c_{1}$ & $m_{\Delta}^{0}$ & $c_{1}$ & $m_{\Delta}^{0}$ & $c_{1}$ & $m_{\Delta}^{0}$ & $c_{1}$ & $m_{\Delta}^{0}$ & $c_{1}$ \\
\hline $\mathcal{O}\left(p^{3}\right) \quad \mathrm{HB} \chi \mathrm{PT}$ & $1.248(31)$ & $-1.19(4)$ & $1.222(68)^{*}$ & $-1.20(5)^{*}$ & $1.241(27)$ & $-1.21(4)$ & $1.274(33)$ & $-1.17(4)$ & $1.251(16)$ & $-1.20(2)$ \\
\hline $\begin{array}{l}\mathcal{O}\left(p^{4} / \Delta\right) \delta \text {-scheme } \\
\Delta^{+, 0}\end{array}$ & $1.258(126)$ & $-1.15(43)$ & & & $1.347(90)$ & $-0.85(30)$ & $1.267(80)$ & $-1.16(20)$ & $1.261(54)$ & $-1.16(17)$ \\
\hline $\mathcal{O}\left(p^{3}\right) \quad \mathrm{HB} \chi \mathrm{PT}$ & $1.255(40)$ & $-1.20(5)$ & $1.261(6)^{*}$ & $-1.19(8)^{*}$ & $1.256(33)$ & $-1.20(4)$ & $1.264(32)$ & $-1.18(4)$ & $1.262(19)$ & $-1.19(3)$ \\
\hline $\mathcal{O}\left(p^{4} / \Delta\right) \delta$-scheme & $1.302(43)^{*}$ & $-1.03(7)^{*}$ & & & $1.372(104)$ & $-0.81(32)$ & $1.373(65)$ & $-0.85(17)$ & $1.267(42)$ & $-1.16(12)$ \\
\hline
\end{tabular}

determined from the nucleon fit we find at the physical point $\sigma_{N}=66.7 \pm 1.3 \mathrm{MeV}$, where the error is statistical. This value is larger than the prevailing value of $45 \pm$ $8 \mathrm{MeV}$ [47] but in agreement with a new analysis [48] that includes additional data. Our current calculation does not include a dynamical strange quark and a better understanding of this term could come when simulations with dynamical strange quarks are available [49]. Note that given the role of the sigma term for what concerns the chiral extrapolation as well as its implication in dark matter detection [50] it is clear that a serious effort to better fix its experimental value is highly desirable.

Chiral corrections to the nucleon mass are known to $\mathcal{O}\left(p^{4}\right)$ within several expansion schemes. In $\mathrm{HB} \chi \mathrm{PT}$ to $\mathcal{O}\left(p^{4}\right)$ with dimensional regularization $[43,51,52]$ the result is given by

$$
\begin{aligned}
m_{N}= & m_{N}^{0}-4 c_{1} m_{\pi}^{2}-\frac{3 g_{A}^{2}}{32 \pi f_{\pi}^{2}} m_{\pi}^{3}-4 E_{1}(\lambda) m_{\pi}^{4} \\
& +\frac{3 m_{\pi}^{4}}{32 \pi^{2} f_{\pi}^{2}}\left[\frac{1}{4}\left(c_{2}-\frac{2 g_{A}^{2}}{m_{N}^{0}}\right)\right. \\
& \left.-\left(c_{2}-8 c_{1}+4 c_{3}+\frac{g_{A}^{2}}{m_{N}^{0}}\right) \log \left(\frac{m_{\pi}}{\lambda}\right)\right] .
\end{aligned}
$$

We take the cutoff scale $\lambda=1 \mathrm{GeV}$ and fix the dimension two low energy constants $c_{2}=3.2 \mathrm{GeV}^{-1}$ [53] and $c_{3}=$ $-3.45 \mathrm{GeV}^{-1}$ [45,52]. This value is consistent with empirical nucleon-nucleon phase shifts $[54,55]$. The counterterm $E_{1}$ is taken as an additional fit parameter. $\mathrm{HB} \chi \mathrm{PT}$ with dimensional regularization is in agreement with covariant baryon $\chi \mathrm{PT}$ with infrared regularization up to a recoil term given by $\frac{3 g_{A}^{2} m_{\pi}^{5}}{256 \pi f_{\pi}^{2} m_{N}^{0}}$ that is of no numerical significance [45]. We have included this term in our fits. In the so-called small scale expansion (SSE) [45], the
$\Delta$-degrees of freedom are explicitly included in covariant baryon $\chi \mathrm{PT}$ by introducing as an additional counting parameter the $\Delta$-nucleon mass splitting, $\Delta \equiv m_{\Delta}-m_{N}$, taking $\mathcal{O}\left(\Delta / m_{N}\right) \sim \mathcal{O}\left(m_{\pi} / m_{N}\right)$. In SSE the nucleon mass is given by

$$
\begin{aligned}
m_{N}= & m_{N}^{0}-4 c_{1} m_{\pi}^{2}-\frac{3 g_{A}^{2}}{32 \pi f_{\pi}^{2}} m_{\pi}^{3}-4 E_{1}(\lambda) m_{\pi}^{4} \\
& -\frac{3\left(g_{A}^{2}+3 c_{A}^{2}\right)}{64 \pi^{2} f_{\pi}^{2} m_{N}^{0}} m_{\pi}^{4}-\frac{\left(3 g_{A}^{2}+10 c_{A}^{2}\right)}{32 \pi^{2} f_{\pi}^{2} m_{N}^{0}} m_{\pi}^{4} \log \left(\frac{m_{\pi}}{\lambda}\right) \\
& -\frac{c_{A}^{2}}{3 \pi^{2} f_{\pi}^{2}}\left(1+\frac{\Delta}{2 m_{N}^{0}}\right)\left[\frac{\Delta}{4} m_{\pi}^{2}+\left(\Delta^{3}-\frac{3}{2} m_{\pi}^{2} \Delta\right)\right. \\
& \left.\times \log \left(\frac{m_{\pi}}{2 \Delta}\right)+\left(\Delta^{2}-m_{\pi}^{2}\right) R\left(m_{\pi}\right)\right],
\end{aligned}
$$

where $R\left(m_{\pi}\right)=\sqrt{m_{\pi}^{2}-\Delta^{2}} \cos ^{-1}\left(\frac{\Delta}{m_{\pi}}\right)$ if $m_{\pi}>\Delta$ and $R\left(m_{\pi}\right)=\sqrt{\Delta^{2}-m_{\pi}^{2}} \log \left(\frac{\Delta}{m_{\pi}}+\sqrt{\frac{\Delta^{2}}{m_{\pi}^{2}}-1}\right)$ for $m_{\pi} \leq \Delta$. We take $c_{A}=1.127$ [45], $\lambda=1 \mathrm{GeV}$ and fit the counterterm $E_{1}$. A different counting scheme, known as the $\delta$-scheme, takes $\Delta / m_{N} \sim \mathcal{O}(\delta)$ and $m_{\pi} / m_{N} \sim \mathcal{O}\left(\delta^{2}\right)$ [44]. Using the $\delta$-scheme in a covariant chiral expansion to order $p^{4} / \Delta$ one obtains an expansion that has a similar form for the nucleon and $\Delta$ mass. The nucleon mass is given by

$$
\begin{aligned}
m_{N}= & m_{N}^{0}-4 c_{1} m_{\pi}^{2}-\frac{1}{2} \frac{m_{N}^{0}}{\left(8 \pi f_{\pi}\right)^{2}}\left[9 g_{A}^{2} V_{\text {loop }}\left(\frac{m_{\pi}}{m_{N}^{0}}, 0\right)\right. \\
& \left.+\frac{4 h_{A}^{2}}{\left(1+\frac{\Delta}{m_{N}^{0}}\right)^{2}} V_{\text {loop }}\left(\frac{m_{\pi}}{m_{N}^{0}}, \frac{\Delta}{m_{N}^{0}}\right)\right]+c_{2} m_{\pi}^{4} .
\end{aligned}
$$

The $\pi N$ and $\pi \Delta$ loop function $V_{\text {loop }}$ is given in Ref. [44] and, following the same reference, we take the value of $h_{A}=2.85$. Here we use the variant of the $\delta$-scheme that includes the $\pi \Delta$-loop and adds the fourth order term $c_{2} m_{\pi}^{4}$ 
as an estimate of higher order effects, since the complete fourth order result is not available. The parameter $c_{2}$ is to be determined from the lattice data. The fits using these different formulations are shown in Fig. 11. At $\beta=3.9$ shown in the left panel we used $a_{\beta=3.9}=0.0855 \mathrm{fm}$ to convert to physical units. We have four values for the $24^{3} \times 48$ lattice and one for the larger lattice. At $\beta=$ 4.05 we only use results at the three smallest pion masses since including the result at the largest pion mass yields fits with unacceptably large $\chi^{2} /$ d.o.f. Therefore only at lowest order $\chi \mathrm{PT}$ where we have only two fitting parameters we can perform a fit. For the higher order we give the values of $m_{N}^{0}$ and $c_{1}$ of the curves that pass through all the lattice points. Since cutoff effects are consistent with zero for these two values of $\beta$ we can use these two sets of results in a combined fit. For the lattice data at $\beta=4.05$ we use $a_{\beta=4.0}=0.0667 \mathrm{fm}$ determined from $f_{\pi}$, to convert to physical units. The experimental value of the nucleon is shown with the asterisk. In Table VI we give the values of $m_{N}^{0}$ and $c_{1}$ when simultaneous fits to both $\beta=3.9$ and $\beta=4.05$ data are done. We use the lattice spacings determined from the pion decay constant to convert to physical units [14]. These fits are shown in Fig. 11 when using higher order $\chi \mathrm{PT}$. All formulations provide a good description of the lattice results and yield a nucleon mass at the physical point consistent with the experimental value. As already discussed, the value of the nucleon mass that we find using Eq. (23) is 963(12) MeV. The corresponding value using $\mathcal{O}\left(p^{4}\right) \mathrm{HB} \chi \mathrm{PT}$ is $955(33) \mathrm{MeV}$. We take the difference between these two mean values as an estimate of the systematic error due to the chiral extrapolation and quote $963 \pm 12$ (stat) \pm 8 (syst) $\mathrm{MeV}$ as our prediction of the nucleon mass. Within the statistical and estimated systematical uncertainty this value is close to the experimental one. Furthermore the values extracted for the nucleon at the chiral limit $m_{N}^{0}$ as well as $c_{1}$ are in agreement in all formulations. In addition the value of nucleon $\sigma_{N}$ term defined in Eq. (24) can be evaluated using $\mathrm{HB} \chi \mathrm{PT}$ to $\mathcal{O}\left(p^{4}\right)$ of Eq. (25). If we use the next to leading order relation between $m_{\pi}^{2}$ and the quark mass $\mu_{q}[13,14,17]$ instead of the leading order relation $m_{\pi}^{2} \propto \mu_{q}$ we find a value of $67 \pm 8.0 \mathrm{MeV}$ at the physical point, which is consistent with the value obtained to $\mathcal{O}\left(p^{3}\right)$ albeit with a larger error. We note that the impact on $\sigma_{N}$ of using Eq. (24) with the next to leading order result, rather than the lowest order relation, between $m_{\pi}^{2}$ and $\mu_{q}$ is small and yields a relative decrease of its value at the physical point of about $2 \%$ only.

The consistency between the $\mathcal{O}\left(p^{3}\right)$ result and the higher order expansions allows for an extrapolation to the physical point and a determination of the lattice spacing using the nucleon mass. Fixing the lattice spacing from the nucleon mass allows for a comparison with the value obtained from the pion sector and provides a nontrivial check of our lattice formulation. We consider $a_{\beta=3.9}$ and $a_{\beta=4.05}$ as independent fit parameters in a combined fit of data at $\beta=3.9$ and $\beta=4.05$ where the physical nucleon mass is included with no error. In this way the lattice spacings can be determined solely by using as input the nucleon mass at the physical point. Using the leading oneloop result we find $a_{\beta=3.9}=0.0889(12) \mathrm{fm}$ and $a_{\beta=4.05}=$ $0.0691(10) \mathrm{fm}$. The quality of these fits are shown in Fig. 12. The values of the lattice spacing obtained to $\mathcal{O}\left(p^{4}\right)$ using Eq. (25) are given in Table VII. Both SSE and the $\delta$-scheme defined by Eqs. (26) and (27), which include explicitly $\Delta$-degrees of freedom, yield values that are consistent with those obtained in $\mathrm{HB} \chi \mathrm{PT}$. The variation in the value of $a$ in the different chiral extrapolation schemes gives an estimate of the systematic error involved in the chiral extrapolation. We take the difference between the mean values obtained using $\mathcal{O}\left(p^{3}\right)$ and $\mathcal{O}\left(p^{4}\right) \mathrm{HB} \chi \mathrm{PT}$ as an estimate of the systematic error. Our lattice spacings fixed using the nucleon mass are therefore $a_{\beta=3.9}=$ $0.0889 \pm 0.0012$ (stat) \pm 0.0014 (syst) fm and $a_{\beta=4.05}=$ $0.0691 \pm 0.0010$ (stat) \pm 0.0010 (syst) fm and are on the upper bound of the values obtained using $f_{\pi}$.

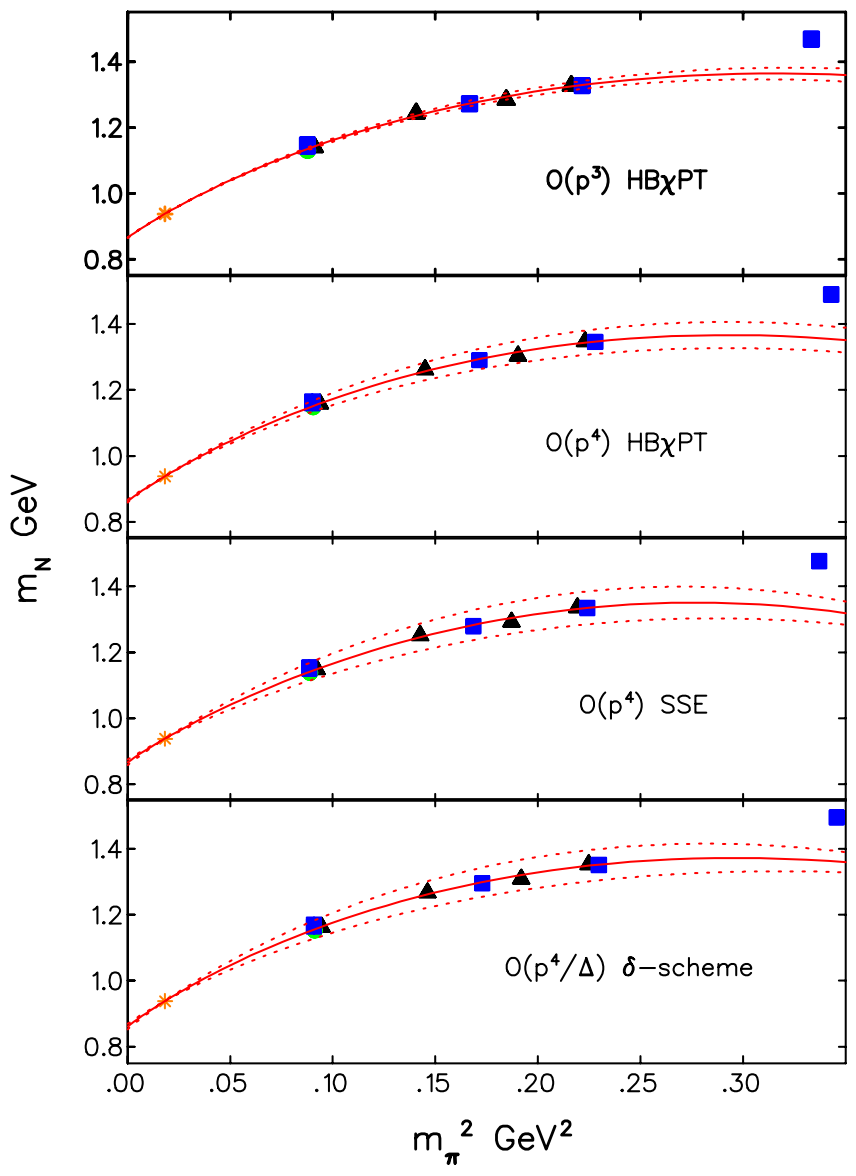

FIG. 12 (color online). Simultaneous chiral fits to the nucleon mass using results at $\beta=3.9$ and $\beta=4.05$ excluding the heaviest pion value. The fits are done so that the physical point shown by the asterisk is reproduced thereby fixing the lattice spacings. The rest of the notation is the same as that of Fig. 11. 
TABLE VII. Determination of the lattice spacing in $\mathrm{fm}$ and $m_{N}^{0}$ in GeV using the nucleon mass. Fitting to the nucleon continuum results obtained by linear interpolation at the five lighter reference pion masses we extract the continuum value of $r_{0}$ in fm by constraining the fits to reproduce the physical nucleon mass.

\begin{tabular}{lccccccccc}
\hline \hline & \multicolumn{3}{c}{$\beta=3.9$} & \multicolumn{2}{c}{$\beta=4.05$} & \multicolumn{3}{c}{$\beta=3.9$ and $\beta=4.05$} & \multicolumn{2}{c}{ Continuum } \\
\hline & $m_{N}^{0}$ & $a_{\beta=3.9}$ & $m_{N}^{0}$ & $a_{\beta=4.05}$ & $m_{N}^{0}$ & $a_{\beta=3.9}$ & $a_{\beta=4.05}$ & $m_{N}^{0}$ & $r_{0}$ \\
$\mathcal{O}\left(p^{3}\right) \mathrm{HB} \chi \mathrm{PT}$ & $0.865(2)$ & $0.0886(18)$ & $0.868(5)$ & $0.0708(37)$ & $0.866(1)$ & $0.0889(12)$ & $0.0691(10)$ & $0.868(2)$ & $0.473(9)$ \\
$\mathcal{O}\left(p^{4}\right) \mathrm{HB} \chi \mathrm{PT}$ & $0.862(9)$ & $0.0869(46)$ & 0.871 & 0.0717 & $0.863(4)$, & $0.0875(26)$ & $0.0681(24)$ & $0.863(8)$ & $0.461(23)$ \\
$\mathcal{O}\left(p^{4}\right) \mathrm{SSE}$ & $0.865(10)$ & $0.0876(47)$ & 0.876 & 0.0724 & $0.866(9)$ & $0.0884(40)$ & $0.0687(31)$ & $0.866(9)$ & $0.464(24)$ \\
$\mathcal{O}\left(p^{4} / \Delta\right) \delta$-scheme & $0.859(11)$ & $0.0865(65)$ & 0.870 & 0.0717 & $0.861(9)$ & $0.0873(39)$ & $0.0678(31)$ & $0.861(10)$ & $0.458(25)$ \\
\hline \hline
\end{tabular}

The physical spatial volumes of the $24^{3}$ lattice at $\beta=$ 3.9 and that of the $32^{3}$ lattice at $\beta=4.05$ are about $(2.1)^{3} \mathrm{fm}^{3}$. Bearing in mind that volume corrections for this lattice size are shown to be small we use results obtained on these two almost equal volumes to estimate our masses at the continuum limit. In order to take the continuum limit we interpolate data, expressed in units of $r_{0}$, at the same value of $r_{0} m_{\pi}$, where the Sommer parameter $r_{0}$ is determined from the force between two static quarks. We use $r_{0} / a=4.46(3), r_{0} / a=5.22(2)$, and $r_{0} / a=6.61(3)$ for $\beta=3.8, \beta=3.9$ and $\beta=4.05$, respectively [7]. The values of $r_{0} m_{\pi}$ that we choose are close to the pion mass values where our computation is done and are given in Table VIII. We use a linear interpolation or the fit curves determined using chiral effective theories to obtain the nucleon mass at these reference values of $r_{0} m_{\pi}$. This procedure is done for our three $\beta$-values. We use the results at constant $r_{0} m_{\pi}$ at $\beta=3.9$ and $\beta=4.05$

TABLE VIII. Results for the nucleon, $\Delta^{++,-}$and $\Delta^{+, 0}$ mass interpolated at the same value of $r_{0} m_{\pi}$ for the three $\beta$ values. The continuum limit is then taken at constant $r_{0} m_{\pi}$ using the results at $\beta=3.9$ and $\beta=4.05$. We give results using linear interpolation in the second, fourth, and sixth columns whereas in the third, fifth, and seventh columns we give the results using lowest order $\mathrm{HB} \chi \mathrm{PT}$.

\begin{tabular}{|c|c|c|c|c|c|c|}
\hline \multirow{2}{*}{$\begin{array}{l}r_{0} m_{\pi} \\
\beta=3 .\end{array}$} & \multicolumn{2}{|c|}{$r_{0} m_{N}$} & \multicolumn{2}{|c|}{$r_{0} m_{\Delta^{++,-}}$} & \multicolumn{2}{|c|}{$r_{0} m_{\Delta^{+, 0}}$} \\
\hline & & & & & & \\
\hline 0.70 & $2.654(95)$ & $2.668(55)$ & $3.596(119)$ & $3.511(69)$ & $3.502(161)$ & $3.528(79)$ \\
\hline 0.80 & $2.804(45)$ & $2.807(41)$ & $3.637(54)$ & $3.614(47)$ & $3.641(65)$ & $3.652(53)$ \\
\hline 0.90 & $2.935(40)$ & $2.933(27)$ & $3.667(75)$ & $3.701(28)$ & $3.790(111)$ & $3.760(30)$ \\
\hline 1.00 & $3.044(45)$ & $3.043(21)$ & $3.731(83)$ & $3.767(31)$ & $3.885(120)$ & $3.850(33)$ \\
\hline 1.10 & $3.133(34)$ & $3.129(31)$ & $3.831(63)$ & $3.804(57)$ & $3.918(72)$ & $3.915(64)$ \\
\hline 1.25 & $3.256(28)$ & $3.201(63)$ & $4.086(62)$ & 3.793(111) & $4.127(64)$ & $3.950(127)$ \\
\hline \multicolumn{7}{|c|}{$\beta=3.9$} \\
\hline 0.70 & $2.666(27)$ & $2.672(17)$ & $3.548(60)$ & $3.481(35)$ & $3.632(88)$ & $3.498(46)$ \\
\hline 0.80 & $2.840(27)$ & $2.809(13)$ & $3.621(65)$ & $3.614(25)$ & $3.641(61)$ & $3.633(32)$ \\
\hline 0.90 & $2.947(22)$ & $2.933(12)$ & $3.720(56)$ & $3.734(16)$ & $3.725(52)$ & $3.754(20)$ \\
\hline 1.00 & $3.013(31)$ & $3.041(14)$ & $3.841(60)$ & $3.837(18)$ & $3.861(46)$ & $3.859(17)$ \\
\hline 1.10 & $3.132(24)$ & $3.125(20)$ & $3.917(50)$ & $3.916(30)$ & $3.943(50)$ & $3.938(31)$ \\
\hline 1.25 & $3.313(76)$ & $3.194(33)$ & $4.028(66)$ & $3.976(56)$ & 4.061(105) & $4.000(62)$ \\
\hline \multicolumn{7}{|c|}{$\beta=4.05$} \\
\hline 0.70 & $2.676(56)$ & $2.665(47)$ & $3.565(124)$ & $3.428(81)$ & $3.550(97)$ & $3.477(71)$ \\
\hline 0.80 & $2.843(71)$ & $2.795(36)$ & $3.627(137)$ & $3.571(59)$ & $3.627(107)$ & $3.609(51)$ \\
\hline 0.90 & $2.903(40)$ & $2.910(24)$ & $3.669(45)$ & $3.701(35)$ & $3.690(45)$ & $3.727(30)$ \\
\hline 1.00 & $3.003(40)$ & $3.007(16)$ & $3.771(45)$ & $3.815(18)$ & $3.793(46)$ & $3.827(18)$ \\
\hline 1.10 & $3.086(24)$ & $3.079(19)$ & $3.932(43)$ & $3.905(35)$ & $3.936(50)$ & $3.902(37)$ \\
\hline 1.25 & $3.287(24)$ & $3.126(42)$ & 4.017(48) & $3.982(85)$ & $4.038(49)$ & $3.953(84)$ \\
\hline \multicolumn{7}{|c|}{ Continuum } \\
\hline 0.70 & $2.667(24)$ & $2.671(16)$ & $3.551(54)$ & $3.472(32)$ & $3.595(65)$ & $3.492(38)$ \\
\hline 0.80 & $2.840(25)$ & $2.807(12)$ & $3.622(59)$ & $3.608(23)$ & $3.638(53)$ & $3.626(27)$ \\
\hline 0.90 & 2.936(19) & $2.929(11)$ & $3.687(35)$ & $3.728(15)$ & $3.705(34)$ & $3.746(16)$ \\
\hline 1.00 & $3.009(25)$ & $3.025(11)$ & $3.794(36)$ & $3.826(13)$ & $3.827(33)$ & $3.844(12)$ \\
\hline 1.10 & $3.109(17)$ & $3.101(14)$ & $3.926(33)$ & $3.916(23)$ & $3.939(35)$ & $3.924(24)$ \\
\hline 1.25 & $3.289(23)$ & $3.168(26)$ & 4.021(39) & $3.978(47)$ & $4.042(44)$ & $3.984(50)$ \\
\hline
\end{tabular}


to estimate the continuum limit by fitting to a constant under the assumption that residual cutoff effects on the pion and nucleon masses as well as on $r_{0}$ at $\beta=3.9$ and $\beta=4.05$ are negligible. This assumption is corroborated by our lattice data shown in Fig. 9. Results at $\beta=3.8$ at the same value of $r_{0} m_{\pi}$ serve as a check for the consistency of this procedure. This is illustrated in Fig. 13 where results at $\beta=3.8$ are consistent with the constant fit. The results at the continuum limit are then chirally extrapolated. The parameters obtained are given in Table VI and the fits are shown in Fig. 14 where we excluded the heaviest pion mass from these fits. The values of $m_{N}^{0}$ and $c_{1}$ obtained from the fits to continuum results are consistent with the values obtained using results at finite $a$. This demonstrates that cutoff effects are small.

The value of the parameter $r_{0}$ can be determined from our results in the continuum limit using the value of the physical nucleon mass. We give the extracted values in Table VII where we used linear interpolation to obtain the nucleon mass at the reference values of $r_{0} m_{\pi}$. The values extracted for $r_{0}$ in the continuum limit using these fits are consistent. Had we used chiral fits at $\mathcal{O}\left(p^{3}\right)$ to interpolate the value extracted would change by $0.004 \mathrm{fm}$ and at $\mathcal{O}\left(p^{4}\right)$ by $0.002 \mathrm{fm}$. These changes are smaller than the statistical

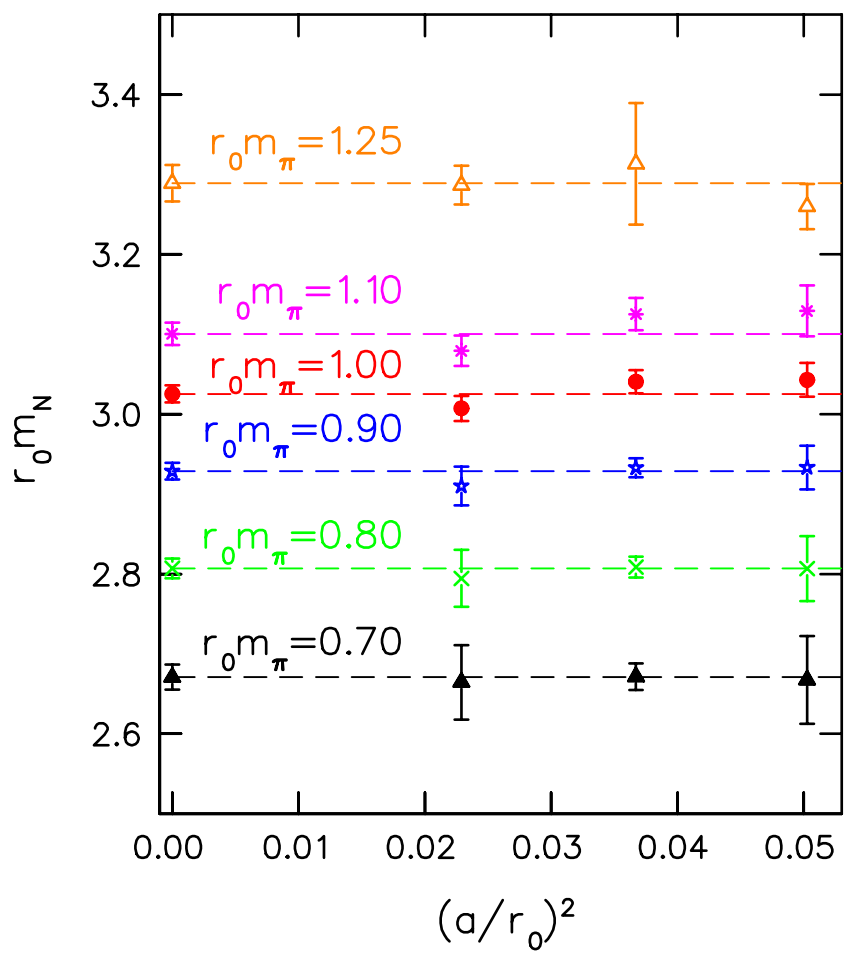

FIG. 13 (color online). Continuum limit of the nucleon mass using the lowest order $\mathrm{HB} \chi \mathrm{PT}$ to interpolate except for the heaviest pion mass where we used linear interpolation. For $r_{0}$ and $a$ we use the values determined in the pion sector, namely $r_{0} / a=4.46(3), \quad\left(r_{0}=0.444(4) \mathrm{fm}\right), \quad r_{0} / a=5.22(2), \quad\left(r_{0}=\right.$ $0.446(3) \mathrm{fm})$ and $r_{0} / a=6.61(3),\left(r_{0}=0.441(4) \mathrm{fm}\right)$ at $\beta=$ 3.8, $\beta=3.9$, and $\beta=4.05$, respectively [7].

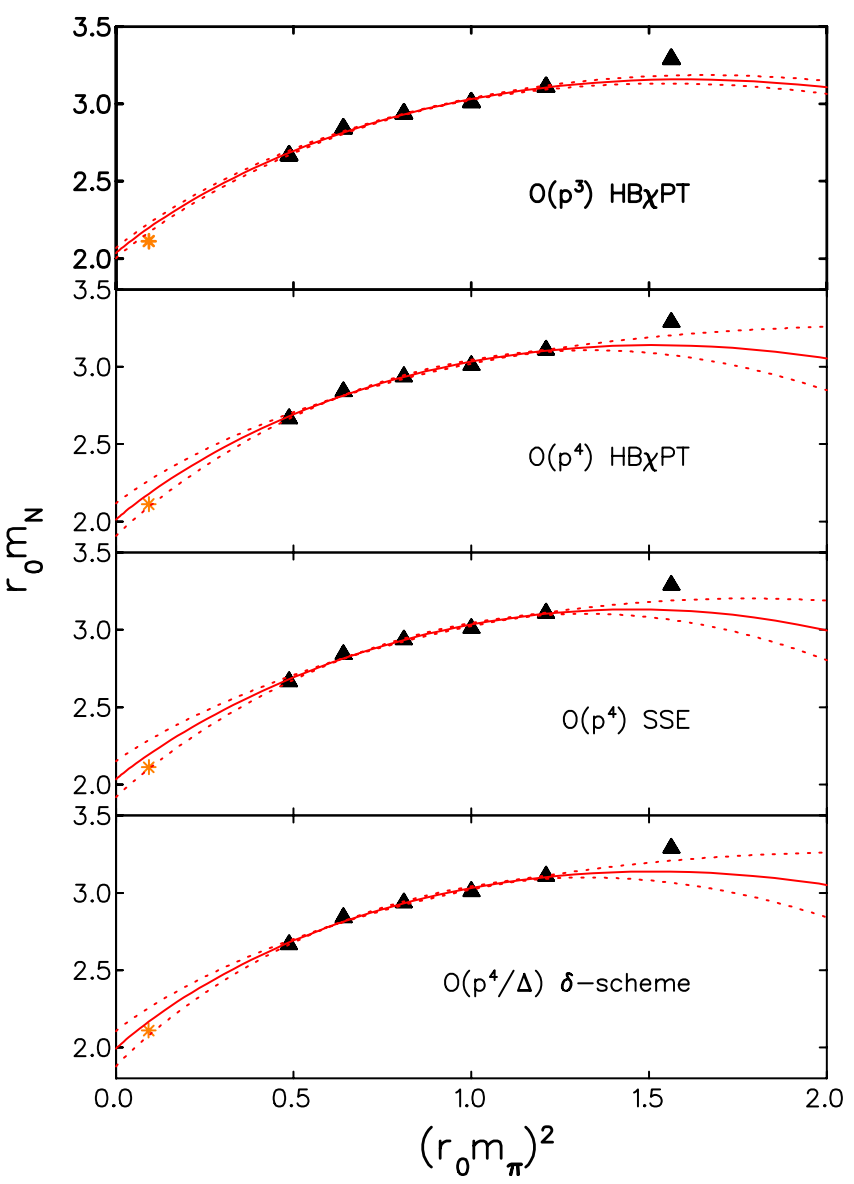

FIG. 14 (color online). Chiral fits to the nucleon mass after extrapolating to the continuum limit at fixed $r_{0} m_{\pi}$ using linear interpolation, i.e. the data given in the second column of Table VIII. The fits were done excluding the heaviest pion mass. The asterisk shows the physical point using the estimated continuum value of $r_{0}=0.444 \mathrm{fm}$.

errors. We again take the variation in the value of $r_{0}$ at $\mathcal{O}\left(p^{3}\right)$ and $\mathcal{O}\left(p^{4}\right) \mathrm{HB} \chi \mathrm{PT}$ as an estimate of the systematic error due to the chiral extrapolation. Using the values given in Table VII this difference is $0.012 \mathrm{fm}$. We add to this error the variation in the values obtained using a linear interpolation scheme and the $\mathcal{O}\left(p^{3}\right)$ fit, which is $0.004 \mathrm{fm}$. Therefore the value that we find is $r_{0}=0.473 \pm$ 0.09 (stat) \pm 0.016 (syst) fm. This value of $r_{0}$, like for the lattice spacing, is at the upper bound of the value $r_{0}=$ $0.444(3) \mathrm{fm}$ [14] determined using $f_{\pi}$. The validation of these consistency checks suggests that lattice artifacts that can affect the value of the lattice spacing when using different observables are small.

\section{B. $\Delta$ mass}

We perform a similar analysis as for the nucleon mass in the case of the $\Delta$.

The leading one-loop $\mathrm{HB} \chi \mathrm{PT}$ result in the case of the $\Delta$ mass has the same form as that for the nucleon mass and is 
given by

$$
m_{\Delta}=m_{\Delta}^{0}-4 c_{1} m_{\pi}^{2}-\frac{25}{81} \frac{3 H_{A}^{2}}{32 \pi f_{\pi}^{2}} m_{\pi}^{3},
$$

where $m_{\Delta}^{0}$ is the $\Delta$ mass at the chiral limit and $c_{1}$ now denotes the coefficient of the $m_{\pi}^{2}$-term for the $\Delta$ mass. For the $\Delta$ axial coupling, $H_{A}$, we use the $\mathrm{SU}(6)$ relation $H_{A}=$ $(9 / 5) g_{A}$ and therefore the one-loop contribution takes the same numerical value as in the nucleon case. We also consider the $\delta$-scheme to order $\mathcal{O}\left(p^{4} / \Delta\right)$ which yields an expression that is similar to the nucleon case:

$$
\begin{aligned}
m_{\Delta}= & m_{\Delta}^{0}-4 c_{1} m_{\pi}^{2}-\frac{1}{2} \frac{m_{\Delta}^{0}}{\left(8 \pi f_{\pi}\right)^{2}}\left[g_{A}^{2} V_{\text {loop }}\left(\frac{m_{\pi}}{m_{\Delta}^{0}}, 0\right)\right. \\
& \left.+4 h_{A}^{2} V_{\text {loop }}\left(\frac{m_{\pi}}{m_{\Delta}^{0}}, \frac{\Delta}{m_{\Delta}^{0}}\right)\right]+c_{2} m_{\pi}^{4} .
\end{aligned}
$$

The fits using the $\mathcal{O}\left(p^{3}\right) \mathrm{HB} \chi \mathrm{PT}$ result at $\beta=3.9$ and $\beta=4.05$ are shown in Fig. 15 for the $\Delta^{++,-}$and $\Delta^{+, 0}$ masses using the lattice spacings determined from $f_{\pi}$. It is useful to chirally extrapolate the $\Delta$ mass to see how close current results are to $\Delta(1232)$ taking the lattice spacings as determined from the nucleon mass. The fits in this case are shown in Fig. 16 again using the $\mathcal{O}\left(p^{3}\right) \mathrm{HB} \chi \mathrm{PT}$ result. Agreement with the experimental value of the $\Delta$ mass is better when one uses the lattice spacing determined from the nucleon mass. This indicates that for baryonic observables it is favorable to use the lattice spacing determined from the nucleon mass. We give the values of the parameters that we extract in Table VI.

The continuum extrapolation is carried out as in the nucleon case. We show in Fig. 17 the results for the three different lattice spacings. As in the case of the nucleon, the continuum limit found by averaging results from $\beta=3.9$

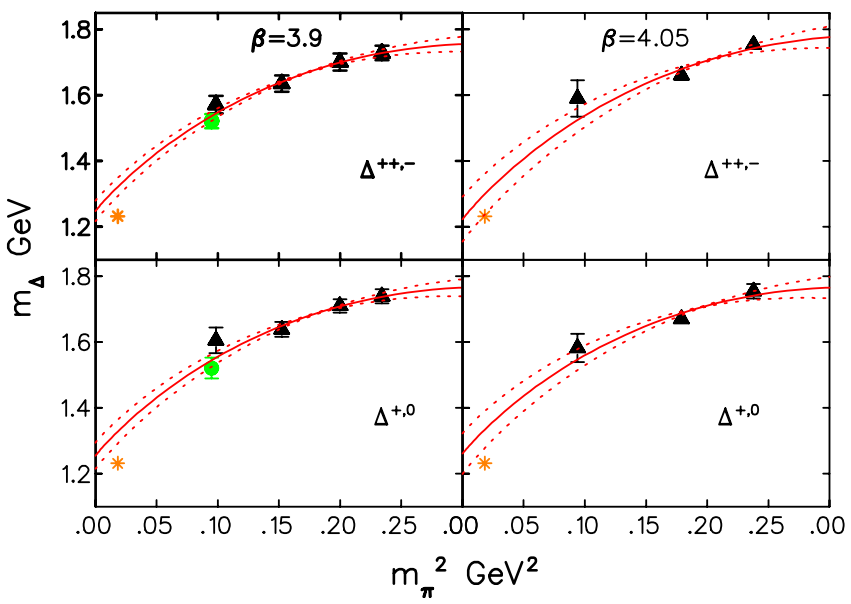

FIG. 15 (color online). Chiral fits to the $\Delta^{++,-}$and $\Delta^{+, 0}$ mass using Eq. (28) taking $a_{\beta=3.9}=0.0855 \mathrm{fm}$ and $a_{\beta=4.05}=$ $0.0667 \mathrm{fm}$ determined from $f_{\pi}$. Filled triangles show results on a $L_{s}=2.1 \mathrm{fm}$. The result on the $2.7 \mathrm{fm}$ volume at $\beta=3.9$ is shown with the filled circle.

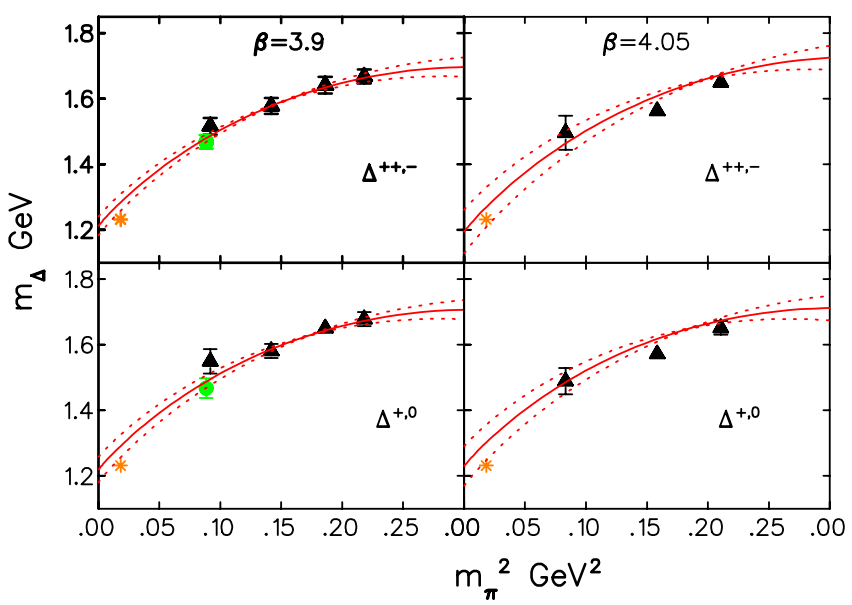

FIG. 16 (color online). Chiral fits to the $\Delta^{++,-}$and $\Delta^{+, 0}$ mass using Eq. (28) with the lattice spacing determined from the nucleon mass. The physical point shown by the asterisk is not included in the fits.

and $\beta=4.05$ is consistent with results at $\beta=3.8$. Furthermore, we find that in the continuum limit $\Delta^{++,-}$ and $\Delta^{+, 0}$ are degenerate within errors, a result that corroborates the absence of isospin breaking. We therefore perform simultaneous fits to both $\Delta^{++,-}$and $\Delta^{+, 0}$ mass using our continuum limit results at the five smallest pion reference masses. These fits using leading chiral perturbation

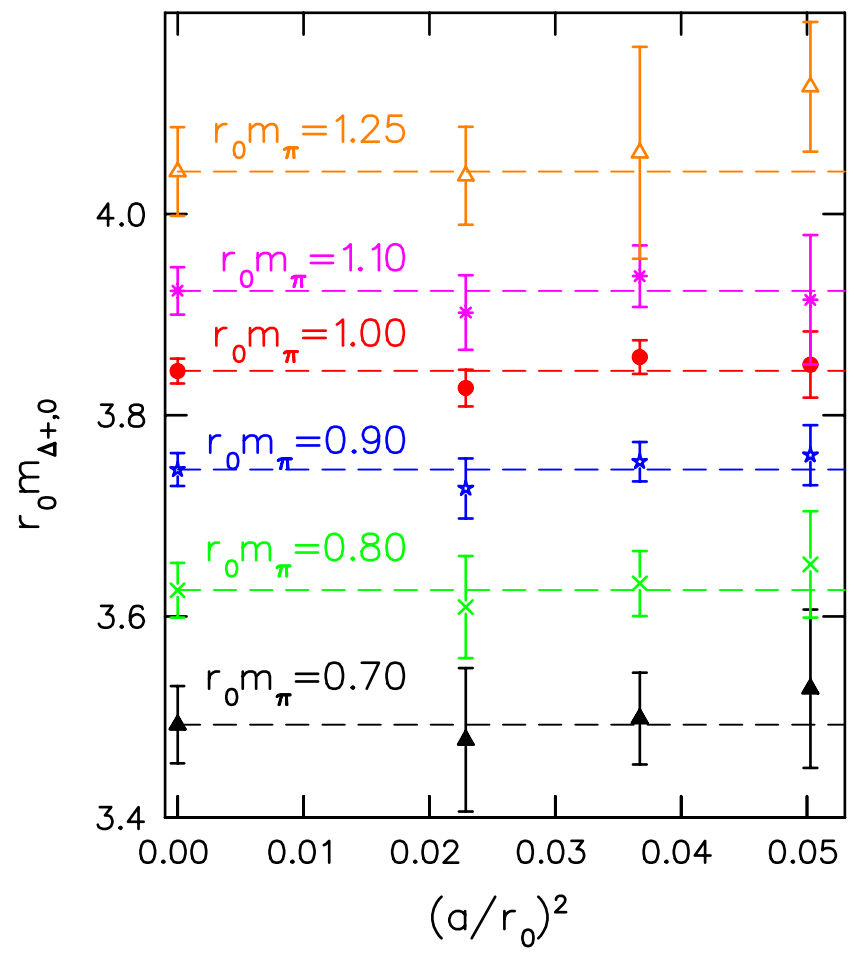

FIG. 17 (color online). Continuum limit of the $\Delta^{+, 0}$ mass using the lowest order $\mathrm{HB} \chi \mathrm{PT}$ to interpolate except for the heaviest pion mass where we use linear interpolation. 


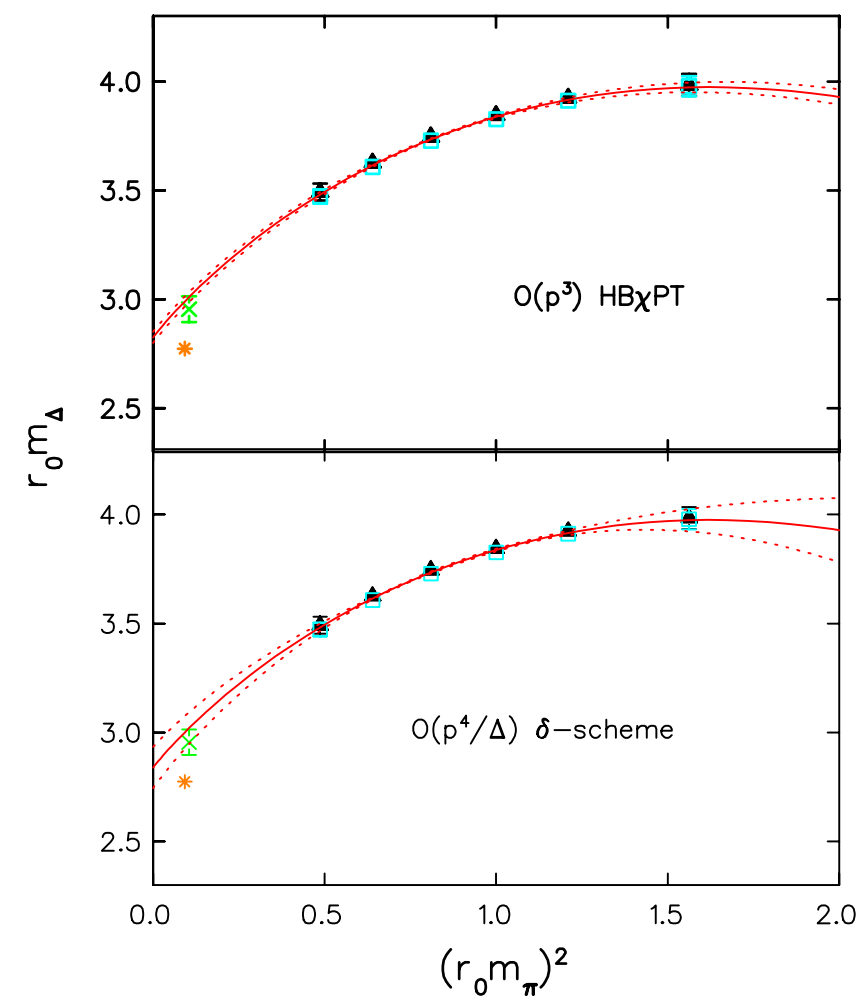

FIG. 18 (color online). Simultaneous chiral fits to the $\Delta^{++,-}$ and $\Delta^{+, 0}$ mass after extrapolating to the continuum limit at fixed $r_{0} m_{\pi}$ excluding the heaviest pion mass. The asterisk denotes the physical $\Delta$ mass using the $r_{0}=0.444(3) \mathrm{fm}$ extracted in the pion sector and it is not included in the fits. The cross denotes the physical point when we use the value of $r_{0}$ extracted from the nucleon sector with only statistical errors.

theory and the $\delta$-scheme are shown in Fig. 18. The physical point is again not included in the fits. We find a $\Delta$ mass at the physical point that is very close to experiment. Again, this agreement improves when we use the value of $r_{0}$ fixed from the nucleon mass. The values that we find for $m_{\Delta}^{0}$ and $c_{1}$ from these simultaneous fits using the continuum results are in good agreement with the values extracted for $\Delta^{++,-}$ and $\Delta^{+, 0}$ at finite lattice spacing. This points to small cutoff effects and to isospin breaking effects that are smaller than statistical errors.

\section{CONCLUSIONS}

Using dynamical twisted mass fermions we obtain accurate results on the nucleon mass for pion masses in the range of 300-500 MeV. The quality of these results allows a chiral extrapolation using heavy baryon chiral perturbation theory to $\mathcal{O}\left(p^{3}\right)$. There is agreement among all approaches for this lowest order result. Performing a simultaneous fit to our results at the two finer lattice spacings we find a value of the nucleon mass of $0.963 \pm$ 0.012 (stat) \pm 0.008 (syst) $\mathrm{GeV}$ where the systematic error is the difference between the mean values obtained at
$\mathcal{O}\left(p^{3}\right)$ and $\mathcal{O}\left(p^{4}\right) \mathrm{HB} \chi \mathrm{PT}$. Comparing results at our three $\beta$-values and at the continuum limit we confirm that cutoff effects are small. Given that this leading one-loop result in $\mathrm{HB} \chi \mathrm{PT}$ yields good fits to our lattice data we use it to extract the lattice spacing from the nucleon mass at the physical point. We find $a_{\beta=3.9}=0.0889 \pm 0.0012$ (stat) \pm 0.0014 (syst) fm and $a_{\beta=4.05}=0.0691 \pm 0.0010$ (stat) \pm 0.0010 (syst) fm. Again the systematic errors are estimated by comparing the value obtained at lowest order to the results obtained using the $\mathcal{O}\left(p^{4}\right)$ in $\mathrm{HB} \chi \mathrm{PT}$. Within this estimated uncertainty of the chiral extrapolation, the value we find for the lattice spacings $a_{\beta=3.9}$ and $a_{\beta=4.05}$ is consistent with the value determined from $f_{\pi}$. A combined analysis of data in the pion and nucleon sector is a promising option that will be considered in the future. We use continuum extrapolated results to determine also the value of $r_{0}$ using the nucleon mass at the physical point. We find a value of $r_{0}=0.473 \pm 0.09$ (stat) \pm 0.016 (syst) fm which is, within errors, consistent with the value determined from $f_{\pi}$. The confirmation that isospin breaking in the $\Delta$ is consistent with zero is a very important conclusion of this work. This is demonstrated by evaluating the mass splitting in the $\Delta$ isospin multiplets for three lattice spacings on two volumes. Consequently the mass of the $\Delta^{++,-}$ and $\Delta^{+, 0}$ obtained in the continuum limit are the same within statistical uncertainties.

The reliable determination of the lattice spacing from the nucleon mass as well as the fact that isospin breaking is consistent with zero for these lattices paves the way for further applications of twisted mass fermions in the baryon sector.

\section{ACKNOWLEDGMENTS}

We thank all other members of the ETM Collaboration for very valuable discussions and for a most enjoyable and fruitful collaboration. We also thank Th. Hemmert for his comments on the nucleon $\sigma$-term. G. K. is supported by the Cyprus Research Promotion Foundation under Contract No. ПENEK/ENI $\Sigma X / 0505-39$. This work is supported in part by the DFG Sonderforschungsbereich/Transregio SFB/TR9-03, DFG Project No. JA 674/5-1, and the EU Integrated Infrastructure Initiative Hadron Physics (I3HP) under Contract No. RII3-CT-2004-506078. R. F. acknowledges MIUR (Italy) for partial financial support under the Contracts No. PRIN04 and No. PRIN06. The analysis runs were performed in the computing centers of the CRI (ParisSud), the CCIN2P3 $\left(\mathrm{IN}_{2} \mathrm{P}_{3}\right)$, the CCRT (CEA). This work was partly done with the help of the "Project ANR-NT053-43577," which is a nonthematic project named QCDNEXT and funding received from the Cyprus Research Promotion Foundation under Contract No. EPYAN/0506/08. Computer time for this project is made available to us by the John von Neumann Institute for Computing on the JUMP and Jubl systems in Jülich and apeNEXT system in Zeuthen, by the UKQCD 
Collaboration on the QCDOC machine at Edinburgh, by INFN and CNRS on the apeNEXT systems in Rome, by BSC on MareNostrum in Barcelona, and by the Leibniz
Computer Center on the Altix system in Munich. We thank these Computer Centers and their staff for technical advice and help.
[1] R. Frezzotti, P. A. Grassi, S. Sint, and P. Weisz (Alpha), J. High Energy Phys. 08 (2001) 058.

[2] R. Frezzotti and G. Rossi, J. High Energy Phys. 48 (2004) 007.

[3] K. Jansen et al., Phys. Lett. B 619, 184 (2005).

[4] F. Farchioni et al., Eur. Phys. J. C 39, 421 (2005).

[5] F. Farchioni et al., Eur. Phys. J. C 47, 453 (2006).

[6] K. Cichy, J. G. Lopez, K. Jansen, A. Kujawa, and A. Shindler, arXiv:0802.3637 [Nucl. Phys. B (to be published)].

[7] P. Dimopoulos, R. Frezzotti, G. Herdoiza, C. Urbach, and U. Wenger (ETM Collaboration), Proc. Sci., LAT2007 (2007) 102 [arXiv:0710.2498].

[8] C. Urbach, K. Jansen, A. Shindler, and U. Wenger, Comput. Phys. Commun. 174, 87 (2006).

[9] K. Jansen, A. Shindler, C. Urbach, and U. Wenger, Proc. Sci., LAT2005 (2006) 118 [arXiv:hep-lat/0510064].

[10] T. Chiarappa, R. Frezzotti, and C. Urbach, Proc. Sci., LAT2005 (2006) 103 [arXiv:hep-lat/0509154].

[11] T. Chiarappa et al., Eur. Phys. J. C 50, 373 (2007).

[12] K. Jansen et al., Proc. Sci., LAT2007 (2007) 036 [arXiv:0709.4434].

[13] P. Boucaud et al. (ETM Collaboration), Phys. Lett. B 650, 304 (2007).

[14] C. Urbach, Proc. Sci., LAT2007 (2007) 022.

[15] H. Leutwyler, arXiv:0706.3138.

[16] S. Simula (ETM Collaboration), Proc. Sci., LAT2007 (2007) [arXiv:0710.0097].

[17] M. Foster and C. Michael (UKQCD Collaboration), Phys. Rev. D 59, 074503 (1999).

[18] C. McNeile and C. Michael (UKQCD Collaboration), Phys. Rev. D 73, 074506 (2006).

[19] R. Frezzotti and G. C. Rossi, Nucl. Phys. B, Proc. Suppl. 128, 193 (2004).

[20] R. Frezzotti and G. C. Rossi, Nucl. Phys. B, Proc. Suppl. 153, 250 (2006).

[21] A. M. Abdel-Rehim, R. Lewis, R. M. Woloshyn, and J. M. S. Wu, Eur. Phys. J. A 31, 773 (2007).

[22] A. M. Abdel-Rehim, R. Lewis, R. M. Woloshyn, and J. M. S. Wu, Phys. Rev. D 74, 014507 (2006).

[23] B. Blossier et al. (ETM Collaboration), J. High Energy Phys. 04 (2008) 020.

[24] V. Lubicz, S. Simula, and C. Tarantino, Proc. Sci., LAT2007 (2007) 374 [arXiv:0710.0329].

[25] B. Blossier, G. Herdoiza, and S. Simula, Proc. Sci., LAT2007 (2007) 346 [arXiv:0710.1414].

[26] R. Baron et al. (ETM Collaboration), Proc. Sci., LAT2007 (2007) 153 [arXiv:0710.1580].

[27] C. Alexandrou et al. (ETM Collaboration), Proc. Sci. LAT2007 (2007) 087 [arXiv:0710.1173].
[28] P. Weisz, Nucl. Phys. B212, 1 (1983).

[29] R. Frezzotti and G. Rossi, Proc. Sci., LAT2007 (2007) 277.

[30] A. Shindler, Phys. Rep. 461, 37 (2008).

[31] P. Boucaud et al., arXiv:0803.0224.

[32] R. Frezzotti, G. Martinelli, M. Papinutto, and G. Rossi, J. High Energy Phys. 04 (2006) 038.

[33] S. Gusken, Nucl. Phys. B, Proc. Suppl. 17, 361 (1990).

[34] C. Alexandrou, S. Gusken, F. Jegerlehner, K. Schilling, and R. Sommer, Nucl. Phys. B414, 815 (1994).

[35] M. Albanese et al. (APE Collaboration), Phys. Lett. B 192, 163 (1987).

[36] J. M. Zanotti, D. B. Leinweber, A. G. Williams, J. Zhang, W. Melnitchouk, and S. Choe, Phys. Rev. D 68, 054506 (2003).

[37] U. Wolff (ALPHA Collaboration), Comput. Phys. Commun. 156, 143 (2004).

[38] P. Boucaud et al. (ETM Collaboration) (unpublished).

[39] C. Colangelo, A. Fuhrer, and C. Häfeli, Nucl. Phys. B, Proc. Suppl. 153, 41 (2006).

[40] C. W. Bernard et al. (MILC Collaboration), Phys. Rev. D 64, 054506 (2001).

[41] C. Aubin et al. (MILC Collaboration), Phys. Rev. D 70, 094505 (2004).

[42] J. Gasser, M. Sainio, and A. Svarc, Nucl. Phys. B307, 779 (1988).

[43] V. Bernard, T. Hemmert, and U.-G. Meissner, Phys. Lett. B 622, 141 (2005).

[44] V. Pascalutsa and M. Vanderhaeghen, Phys. Lett. B 636, 31 (2006).

[45] M. Procura et al., Phys. Rev. D 73, 114510 (2006).

[46] M. Procura, T. R. Hemmert, and W. Weise, Phys. Rev. D 69, 034505 (2004).

[47] J. Gasser, H. Leutwyler, and M. Sainio, Phys. Lett. B 253, 252 (1991)

[48] M. M. Pavan, I. I. Strakovsky, R. L. Workman, and R. A. Arndt, PiN Newslett. 16, 110 (2002).

[49] J. Gasser and H. Leutwyler, Phys. Rep. 87, 77 (1982).

[50] J. Ellis, K. Olive, and C. Savage, Phys. Rev. D 77, 065026 (2008).

[51] S. Steininger, U.-G. Meissner, and N. Fettes, J. High Energy Phys. 09 (1998) 008.

[52] V. Bernard, T. Hemmert, and U.-G. Meissner, Nucl. Phys. A732, 149 (2004).

[53] N. Fettes, U.-G. Meissner, and S. Steininger, Nucl. Phys. A640, 199 (1998).

[54] D. R. Entem and R. Machleidt, Phys. Rev. C 66, 014002 (2002).

[55] E. Epelbaum, W. Glockle, and U.-G. Meissner, Nucl. Phys. A747, 362 (2005). 